\title{
Mean first-passage time of surface-mediated diffusion in spherical domains
}

\author{
O. Bénichou, ${ }^{1}$ D. S. Grebenkov, ${ }^{2,3}$ P. E. Levitz, ${ }^{2}$ C. Loverdo, ${ }^{1}$ and R. Voituriez ${ }^{1}$ \\ ${ }^{1}$ Laboratoire de Physique Théorique de la Matière Condensée (UMR 7600), \\ case courrier 121, Université Paris 6, 4 Place Jussieu, 75255 Paris Cedex \\ ${ }^{2}$ Laboratoire de Physique de la Matière Condensée (UMR7643), \\ CNRS - Ecole Polytechnique, F-91128 Palaiseau Cedex France \\ ${ }^{3}$ Laboratoire Poncelet (UMI 2615), CNRS - Independent University of Moscow, \\ Bolshoy Vlasyevskiy Pereulok 11, 119002 Moscow, Russia
}

(Dated: November 5, 2018)

\begin{abstract}
We present an exact calculation of the mean first-passage time to a target on the surface of a $2 \mathrm{D}$ or $3 \mathrm{D}$ spherical domain, for a molecule alternating phases of surface diffusion on the domain boundary and phases of bulk diffusion. The presented approach is based on an integral equation which can be solved analytically. Numerically validated approximation schemes, which provide more tractable expressions of the mean first-passage time are also proposed. In the framework of this minimal model of surface-mediated reactions, we show analytically that the mean reaction time can be minimized as a function of the desorption rate from the surface.
\end{abstract}

PACS numbers:

\section{INTRODUCTION}

The kinetics of many chemical reactions is influenced by the transport properties of the reactants that they involve [1, 2]. In fact, schematically, any chemical reaction requires first that a given reactant A meets a second reactant B. This first reaction step can be rephrased as a search process involving a searcher A looking for a target B. In a very dilute regime, exemplified by biochemical reactions in cells [3] which sometimes involve only a few copies of reactants, the targets B are sparse and therefore hard to find in this search process language. In such reactions, the first step of search for reactants B is therefore a limiting factor of the global reaction kinetics. In the general aim of enhancing the reactivity of chemical systems, it is therefore needed to optimize the efficiency of this first step of search.

Recently, it has been shown that intermittent processes, combining slow diffusion phases with a faster transport, can significantly increase reactions rates [4, 5]. A minimal model demonstrating the efficiency of this type of search, introduced to account for the fast search of target sequences on DNA by proteins [6] is as follows (see also [7-10]). The pathway followed by the protein, considered as a point-like particle, is a succession of $1 \mathrm{D}$ diffusions along the DNA strand (called sliding phases) with diffusion coefficient $D_{1}$ and $3 \mathrm{D}$ excursions in the surrounding solution. The time spent by the protein on DNA during each sliding phase is assumed to follow an exponential law with dissociation rate $\lambda$. In this minimal model, the 3D excursions are uncorrelated in space, which means that after dissociation from DNA, the protein will rebind the DNA at a random position independently of its starting position. Assuming further that the mean duration of such $3 \mathrm{D}$ excursions $\tau_{2}$ is finite, it has been shown that the mean first-passage time at the target can be minimized as a function of $\tau_{1}=\lambda^{-1}$, as soon as the mean time spent in bulk excursions is not too long. Quantitatively, this condition writes in orders of magnitude as $\tau_{2} \leq L^{2} / D_{1}$, and the minimum of the search time is obtained for $\tau_{1} \simeq \tau_{2}$ in the large $L$ limit. Note that in this minimal model, where the time $\tau_{2}$ is supposed to be a fixed exterior parameter, bulk phases are always beneficial in the large $L$ limit (i.e. allow one to decrease the search time with respect to the situation corresponding to $1 \mathrm{D}$ diffusion only).

In many practical situations however, the duration of the fast bulk excursions strongly depends on the geometrical properties of the system [11 14] and cannot be treated as an independent variable as assumed in the mean-field (MF) model introduced above. An important generic situation concerns the case of confined systems [15 17], involving transport of reactive molecules both in the bulk of a confining domain and on its boundary, referred to as surfacemediated diffusion in what follows. This type of problems is met in situations as varied as heterogeneous catalysis [18, 19], or reactions in porous media and in vesicular systems [15, 16, 20]. In all these examples, the duration of bulk excursions is controlled by the return statistics of the molecule to the confining surface, which crucially depends on the volume of the system. This naturally induces strong correlations between the starting and ending points of bulk excursions, and makes the above MF assumption of uncorrelated excursions largely inapplicable in these examples.

At the theoretical level, the question of determining mean first-passage times in confinement has attracted a lot of attention in recent years for discrete random walks [21 25] and continuous processes [26 29]. More precisely, the surface-mediated diffusion problem considered here generalizes the so-called narrow escape problem, which refers to the time needed for a simple Brownian motion in absence of surface diffusion to escape through a small window of an otherwise reflecting domain. This problem has been investigated both in the mathematical [20, 30 32] and physical [33 36] literature, partly due to the challenge of taking into account mixed boundary conditions. The case of 
surface-mediated diffusion brings the additional question of minimizing the search time with respect to the time spent in adsorption, in the same spirit as done for intermittent processes introduced above. The answer to this question is a priori not clear, since the mean time spent in bulk excursions diverges for large confining domains, so that the condition of minimization mentioned previously cannot be taken as granted, even in the large system limit. In this context, first results have been obtained in [37] where, surprisingly enough, it has been found that, even for bulk and surface diffusion coefficients of the same order of magnitude, the reaction time can be minimized, whereas MF treatments (see for instance [34]) predict a monotonic behavior.

Here, we extend the perturbative results of [37] obtained in the small target size limit. Relying on an integral equation approach, we provide an exact solution for the mean FPT, both for $2 \mathrm{D}$ an $3 \mathrm{D}$ spherical domains, and for any spherical target size. We also develop approximation schemes, numerically validated, that provide more tractable expressions of the mean FPT.

\section{THE MODEL}

The surface-mediated process under study is defined as follows. We consider a molecule diffusing in a spherical confining domain of radius $R$ (see figure 1), alternating phases of boundary diffusion (with diffusion coefficient $D_{1}$ ) and phases of bulk diffusion (with diffusion coefficient $D_{2}$ ). The time spent during each one-dimensional phase is assumed to follow an exponential law with dissociation rate $\lambda$. At each desorption event, the molecule is assumed to be ejected at a distance $a$ from the frontier (otherwise it is instantaneously readsorbed). Although formulated for any value of this parameter $a$ smaller than $R$, in most physical situations of real interest $a \ll R$. The target is perfectly absorbing and defined in $2 D$ by the arc $\theta \in[-\epsilon, \epsilon]$, and in $3 D$ by the region of the sphere such that $\theta \in[0, \epsilon]$ where $\theta$ is in this case the elevation angle in standard spherical coordinates. Note that as soon as $\epsilon \neq 0$, the target can be reached either by surface or bulk diffusion. In what follows we calculate the mean first-passage time at the target for an arbitrary initial condition of the molecule.

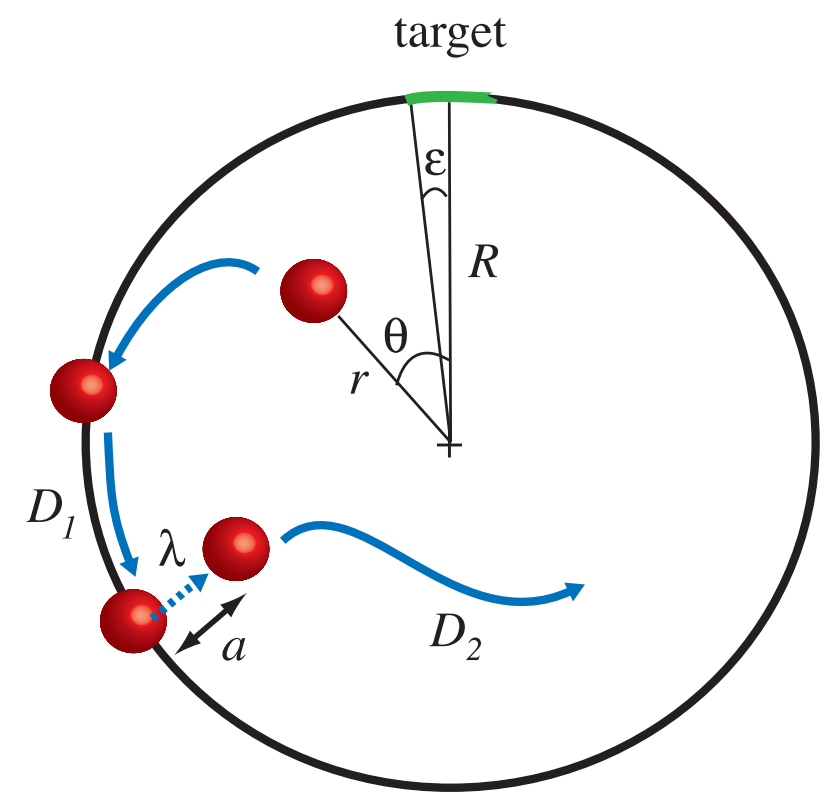

FIG. 1: Model

III. 2D CASE

In this section, the confining domain is a disk of radius $R$ and the target is defined by the arc $\theta \in[-\epsilon, \epsilon]$. 


\section{A. Basic equations}

For the process defined above, the mean first-passage time (MFPT) at the target satisfies the following backward equations

$$
\begin{aligned}
\frac{D_{1}}{R^{2}} t_{1}^{\prime \prime}(\theta)+\lambda\left[t_{2}(R-a, \theta)-t_{1}(\theta)\right] & =-1 \text { for } \theta \in[\epsilon, 2 \pi-\epsilon], \\
D_{2}\left(\frac{\partial^{2}}{\partial r^{2}}+\frac{1}{r} \frac{\partial}{\partial r}+\frac{1}{r^{2}} \frac{\partial^{2}}{\partial \theta^{2}}\right) t_{2}(r, \theta) & =-1,
\end{aligned}
$$

where $t_{1}$ stands for the the MFPT starting from the circle at a position defined on the circumference by the angle $\theta$, and $t_{2}$ for the MFPT starting from the point $(r, \theta)$ within the disk. In these two equations, the first term of the lhs accounts for the diffusion respectively on the circumference and in the bulk, while the second term of Eq. (1) describes desorption events. They have to be completed by two boundary conditions

$$
\begin{aligned}
t_{2}(R, \theta) & =t_{1}(\theta), \\
t_{1}(\theta) & =0 \text { for } \theta \in[0, \epsilon] \cup[2 \pi-\epsilon, 2 \pi],
\end{aligned}
$$

which describe the adsorption events and the absorbing target respectively. Eq.(2) is easily shown to be satisfied by the following Fourier series

$$
t_{2}(r, \theta)=\alpha_{0}-\frac{r^{2}}{4 D_{2}}+\sum_{n=1}^{\infty} \alpha_{n} r^{n} \cos (n \theta)
$$

with unknown coefficients $\alpha_{n}$ to be determined. In particular, we aim at determining the search time $\left\langle t_{1}\right\rangle$, defined as the MFPT, with an initial position uniformly distributed on the boundary of the confining domain. Taking Eq.(5) at $r=R$, we have

$$
\alpha_{0}-\frac{R^{2}}{4 D_{2}}+\sum_{n=1}^{\infty} \alpha_{n} R^{n} \cos (n \theta)= \begin{cases}t_{1}(\theta) & \text { if } \theta \in[\epsilon, 2 \pi-\epsilon] \\ 0 & \text { if } \theta \in[0, \epsilon] \cup[2 \pi-\epsilon, 2 \pi]\end{cases}
$$

so that

$$
\begin{aligned}
\alpha_{0}-\frac{R^{2}}{4 D_{2}} & =\frac{1}{2 \pi} \int_{\epsilon}^{2 \pi-\epsilon} t_{1}(\theta) \mathrm{d} \theta \equiv\left\langle t_{1}\right\rangle, \\
R^{n} \alpha_{n} & =\frac{1}{\pi} \int_{\epsilon}^{2 \pi-\epsilon} t_{1}(\theta) \cos (n \theta) \mathrm{d} \theta \quad(n \geq 1) .
\end{aligned}
$$

In what follows we will make use of the following quantities:

$$
\begin{gathered}
\omega \equiv R \sqrt{\lambda / D_{1}}, \\
x \equiv 1-\frac{a}{R},
\end{gathered}
$$

and

$$
T \equiv \frac{1}{\lambda}+\frac{R^{2}-(R-a)^{2}}{4 D_{2}}
$$

As we proceed to show, two different approaches can be used to solve this problem. (i) The first approach, whose main results have been published in [37], uses the explicit form of the Green function for the two-dimensional problem and relies on a small target size $\epsilon$ expansion. We recall these perturbative results below for the sake of self-consistency and give details of the derivation in Appendix $\mathrm{A}$ (ii) The second approach presented next relies on an integral equation which can be derived for $t_{1}$, and leads to an exact non-perturbative solution. 


\section{B. Perturbative approach}

It is shown in Appendix that the Fourier coefficients of $t_{2}(r, \theta)$ as defined in Eq. (5) satisfy an infinite hierarchy of linear equations, which lead to the following small $\epsilon$ expansion:

$$
\begin{aligned}
& \alpha_{0}=\frac{R^{2}}{4 D_{2}}+\omega^{2} T\left\{\left(2 \sum_{m=1}^{\infty} \frac{1}{\omega^{2}\left(1-x^{m}\right)+m^{2}}\right)-\pi \epsilon+\left(1+2 \omega^{2} \sum_{m=1}^{\infty} \frac{1-x^{m}}{\omega^{2}\left(1-x^{m}\right)+m^{2}}\right) \epsilon^{2}\right\}+\ldots \\
& \alpha_{n}=\frac{\omega^{2} T}{R^{n}\left(\omega^{2}\left(1-x^{n}\right)+n^{2}\right)}\left\{-2+n^{2} \epsilon^{2}+\ldots\right\} .
\end{aligned}
$$

Note that Eq.(11) gives in particular the first terms of the perturbative expansion of the search time $\left\langle t_{1}\right\rangle$ defined in (7) and given in [37]. It should be stressed that since the coefficients of $\epsilon^{k}$ of this expansion diverge with $\omega$, in practice one finds that the range of applicability in $\epsilon$ of this expansion is wider for $\omega$ small.

\section{Integral equation for $t_{1}$}

In this section, we first show that the resolution of the coupled PDEs (11, 2) amounts to solving an integral equation for $t_{1}$ only. As we proceed to show, this integral equation can be solved exactly. Writing Eq. (1) as

$$
\frac{\partial^{2} t_{1}}{\partial \theta^{2}}=-\frac{R^{2}}{D_{1}}-\omega^{2}\left[t_{2}(R-a, \theta)-t_{2}(R, \theta)\right]
$$

and expanding its right-hand side into a Taylor series leads to

$$
\frac{\partial^{2} t_{1}}{\partial \theta^{2}}=-\frac{R^{2}}{D_{1}}-\omega^{2} \sum_{k=1}^{\infty} \frac{(-a)^{k}}{k !}\left(\frac{\partial^{k} t_{2}}{\partial r^{k}}\right)_{R, \theta}
$$

Substituting the Fourier representation (5) for $t_{2}$ into this equation yields

$$
\frac{\partial^{2} t_{1}}{\partial \theta^{2}}=-\frac{R^{2}}{D_{1}}-\omega^{2}\left(\frac{a R}{2 D_{2}}-\frac{a^{2}}{4 D_{2}}\right)-\omega^{2} \sum_{k=1}^{\infty} \frac{(-a)^{k}}{k !} \sum_{n=k}^{\infty} \alpha_{n} n(n-1) \ldots(n-k+1) R^{n-k} \cos (n \theta) .
$$

Changing the order of summations over $n$ and $k$, using the binomial formula and the expression (7) for $\alpha_{n}$ give

$$
\frac{\partial^{2} t_{1}}{\partial \theta^{2}}=-\frac{R^{2}}{D_{1}}-\omega^{2}\left(\frac{a R}{2 D_{2}}-\frac{a^{2}}{4 D_{2}}\right)-\frac{\omega^{2}}{\pi} \sum_{n=1}^{\infty}\left(x^{n}-1\right) \cos (n \theta) \int_{\epsilon}^{2 \pi-\epsilon} \cos \left(n \theta^{\prime}\right) t_{1}\left(\theta^{\prime}\right) \mathrm{d} \theta^{\prime} .
$$

This integro-differential equation for $t_{1}$ can actually easily be transformed into an integral equation for $t_{1}$, by integrating successively two times, which leads to

$$
\begin{aligned}
t_{1}(\theta) & =\frac{1}{2}\left(\frac{R^{2}}{D_{1}}+\omega^{2}\left(\frac{a R}{2 D_{2}}-\frac{a^{2}}{4 D_{2}}\right)\right)(\theta-\epsilon)(2 \pi-\epsilon-\theta) \\
& +\frac{\omega^{2}}{\pi} \sum_{n=1}^{\infty}\left(x^{n}-1\right) \frac{\cos (n \theta)-\cos (n \epsilon)}{n^{2}} \int_{\epsilon}^{2 \pi-\epsilon} \cos \left(n \theta^{\prime}\right) t_{1}\left(\theta^{\prime}\right) \mathrm{d} \theta^{\prime}
\end{aligned}
$$

or equivalently to

$$
\psi(\theta)=(\theta-\epsilon)(2 \pi-\epsilon-\theta)+\Omega \sum_{n=1}^{\infty}\left(x^{n}-1\right) \frac{\cos (n \theta)-\cos (n \epsilon)}{n^{2}} \int_{\epsilon}^{2 \pi-\epsilon} \cos \left(n \theta^{\prime}\right) \psi\left(\theta^{\prime}\right) \mathrm{d} \theta^{\prime}
$$

where

$$
\psi(\theta) \equiv \frac{2 t_{1}(\theta)}{\omega^{2} T}
$$


with $T$ defined in Eq. (10) and $\Omega \equiv \frac{\omega^{2}}{\pi}$. Note that Eq. (17) holds for $\theta \in[\epsilon, 2 \pi-\epsilon]$. When there is no desorption (i.e., $\lambda=0$ ), only the first term in Eq. (18) survives, yielding the classical result [26]

$$
t_{1}(\theta)=\frac{R^{2}}{D_{1}}(\theta-\epsilon)(2 \pi-\epsilon-\theta) .
$$

The same result is obtained for $a=0$, since $x^{n}-1=(1-a / R)^{n}-1=0$. The limit $a=0$ is in fact equivalent to the limit $\lambda=0$ because, after desorption, the particle immediately returns onto the circle $(a=0)$ as if it was never desorbed $(\lambda=0)$.

\section{Exact solution}

Iterating the integral equation (17) shows that the solution $\psi(\theta)$ writes for $\theta \in[\epsilon, 2 \pi-\epsilon]$ :

$$
\psi(\theta)=(\theta-\epsilon)(2 \pi-\epsilon-\theta)+\sum_{n=1}^{\infty} d_{n}[\cos (n \theta)-\cos (n \epsilon)]
$$

with the coefficients $d_{n}$ which satisfy

$$
\sum_{n=1}^{\infty} d_{n}[\cos (n \theta)-\cos (n \epsilon)]=\Omega \sum_{n=1}^{\infty}\left(U_{n}+\sum_{n^{\prime}=1}^{\infty} Q_{n, n^{\prime}} d_{n^{\prime}}\right)[\cos (n \theta)-\cos (n \epsilon)],
$$

where we introduced

$$
\begin{aligned}
& U_{n} \equiv \frac{x^{n}-1}{n^{2}} \int_{\epsilon}^{2 \pi-\epsilon} d \theta^{\prime} \cos \left(n \theta^{\prime}\right)\left(\theta^{\prime}-\epsilon\right)\left(2 \pi-\epsilon-\theta^{\prime}\right)=4 \frac{1-x^{n}}{n^{4}} \xi_{n} \\
& \xi_{n} \equiv(\pi-\epsilon) \cos (n \epsilon)+\frac{\sin (n \epsilon)}{n} \quad(n=1,2, \ldots)
\end{aligned}
$$

and

$$
Q_{n, n^{\prime}} \equiv-\frac{1-x^{n}}{n^{2}} I_{\epsilon}\left(n, n^{\prime}\right) \quad\left(n, n^{\prime}=1,2, \ldots\right)
$$

with

$$
\begin{aligned}
I_{\epsilon}\left(n, n^{\prime}\right) & \equiv \int_{\epsilon}^{2 \pi-\epsilon} \cos (n \theta)\left(\cos \left(n^{\prime} \theta\right)-\cos \left(n^{\prime} \epsilon\right)\right) \mathrm{d} \theta \\
& =\left(1-\delta_{n, n^{\prime}}\right)\left(2 \frac{\cos \left(n^{\prime} \epsilon\right) \sin (n \epsilon)}{n}-\frac{\sin \left(\left(n^{\prime}+n\right) \epsilon\right)}{n^{\prime}+n}-\frac{\sin \left(\left(n^{\prime}-n\right) \epsilon\right)}{n^{\prime}-n}\right) \\
& +\delta_{n, n^{\prime}}\left(\pi-\epsilon+\frac{\sin (2 n \epsilon)}{2 n}\right) \\
& =2\left(1-\delta_{n, n^{\prime}}\right) \frac{\cos (n \epsilon) \frac{\sin \left(n^{\prime} \epsilon\right)}{n^{\prime}}-\cos \left(n^{\prime} \epsilon\right) \frac{\sin (n \epsilon)}{n}}{n^{2}-n^{\prime 2}} n^{\prime 2}+\delta_{n, n^{\prime}}\left(\pi-\epsilon+\frac{\sin (2 n \epsilon)}{2 n}\right) .
\end{aligned}
$$

Since Eq. (21) should be satisfied for any $\theta \in[\epsilon, 2 \pi-\epsilon]$, one gets $\mathbf{d}=\Omega(U+Q \mathbf{d})$, from which

$$
d_{n}=\Omega\left[(I-\Omega Q)^{-1} U\right]_{n} \quad(n=1,2, \ldots) .
$$

Since

$$
(I-\Omega Q)^{-1}=\sum_{i=0}^{\infty}(\Omega Q)^{i},
$$

Eq. (20) with the $d_{n}$ given by Eq. (25) can be seen as a series in powers of $\Omega$, whose $n$-th order coefficient is explicitly written in terms of the $n$-th power of the matrix $Q$. 
Note that the first term in Eq. (20) can also be expended in a Fourier series

$$
\sum_{n=1}^{\infty} e_{n}[\cos (n \theta)-\cos (n \epsilon)]=\left\{\begin{array}{cc}
(\theta-\epsilon)(2 \pi-\epsilon-\theta), & \epsilon<\theta<2 \pi-\epsilon, \\
0, & \text { otherwise }
\end{array}\right.
$$

where the coefficients $e_{n}$ are obtained by multiplying this equation by $\cos m \theta$ and integrating from 0 to $2 \pi$ :

$$
e_{n}=-\frac{4}{\pi n^{2}} \xi_{n} \quad(n=1,2, \ldots) .
$$

Once the $d_{n}$ determined, the search time $\left\langle t_{1}\right\rangle$ is

$$
\left\langle t_{1}\right\rangle \equiv \frac{1}{2 \pi} \int_{0}^{2 \pi} t_{1}(\theta) d \theta=\frac{\omega^{2} T}{4 \pi} \int_{\epsilon}^{2 \pi-\epsilon} \psi(\theta) d \theta=\frac{\omega^{2} T}{2 \pi}\left\{\frac{2}{3}(\pi-\epsilon)^{3}-\sum_{n=1}^{\infty} d_{n} \xi_{n}\right\} .
$$

\section{E. Approximate solution}

While the previous expression of $t_{1}$ is exact, it is not fully explicit, since it requires either the inversion of the matrix $I-\Omega Q$ or the calculation of all the powers of $Q$. We give here an approximation of $(I-\Omega Q)^{-1}$, which in turn provides a convenient and fully explicit representation of $t_{1}$. As shown numerically (see Figs. 2] 3, 4 and section $\nabla$ for more details about numerical methods), this approximation of $t_{1}$ proves to be in quantitative agreement with the exact expression for a wide range of parameters.

This approximation relies on the fact that, in the small target size limit $\epsilon \rightarrow 0$, the matrix $Q$ is diagonal, which mirrors the orthogonality of the $\{\cos (n \theta)\}_{n}$ on $[0,2 \pi]$. More precisely, one has from Eqs. (23]24):

$$
Q_{m, n}=\delta_{m, n} Q_{n, n}+\mathcal{O}\left(\epsilon^{3}\right),
$$

and keeping only the leading term of this expansion yields

$$
d_{n} \approx \Omega\left(1-\Omega Q_{n, n}\right)^{-1} U_{n},
$$

from which we obtain the desired approximation:

$$
\psi(\theta) \approx(\theta-\epsilon)(2 \pi-\epsilon-\theta)+4 \Omega \sum_{n=1}^{\infty}(\cos (n \theta)-\cos (n \epsilon)) \frac{n(\pi-\epsilon) \cos (n \epsilon)+\sin (n \epsilon)}{n^{3}} \frac{1-x^{n}}{n^{2}+\Omega\left(1-x^{n}\right) I_{\epsilon}(n, n)} .
$$

This yields an approximation for the search time:

$$
\left\langle t_{1}\right\rangle \approx \frac{\omega^{2} T}{2 \pi}\left\{\frac{2}{3}(\pi-\epsilon)^{3}-4 \Omega \sum_{n=1}^{\infty} \frac{1-x^{n}}{n^{2}} \frac{\left((\pi-\epsilon) \cos (n \epsilon)+\frac{\sin (n \epsilon)}{n}\right)^{2}}{n^{2}+\Omega\left(1-x^{n}\right)\left(\pi-\epsilon+\frac{\sin (2 n \epsilon)}{2 n}\right)}\right\} .
$$

\section{F. Variations of the search time $\left\langle t_{1}\right\rangle$ with the desorption rate $\lambda$}

In this section, we answer two important questions. When are bulk excursions favorable, meaning enabling to reduce the search time (with respect to the situation with no bulk excursion corresponding to $\lambda=0$ )? If so, is there an optimal value of the desorption rate $\lambda$ minimizing the search time?

\section{When are bulk excursions beneficial to the search?}

This question can be investigated by studying the sign of the derivative $\frac{\partial\left\langle t_{1}\right\rangle}{\partial \lambda}$ at $\lambda=0$. The mean search time from Eq. (29) can also be written as

$$
\left\langle t_{1}\right\rangle=\frac{R^{4}}{2 \pi^{2} D_{1}^{2}}(1+\lambda \eta)\left[\frac{2 \pi D_{1}}{3 R^{2}}(\pi-\epsilon)^{3}-\lambda\left(\xi \cdot(I+\lambda \tilde{Q})^{-1} U\right)\right]
$$



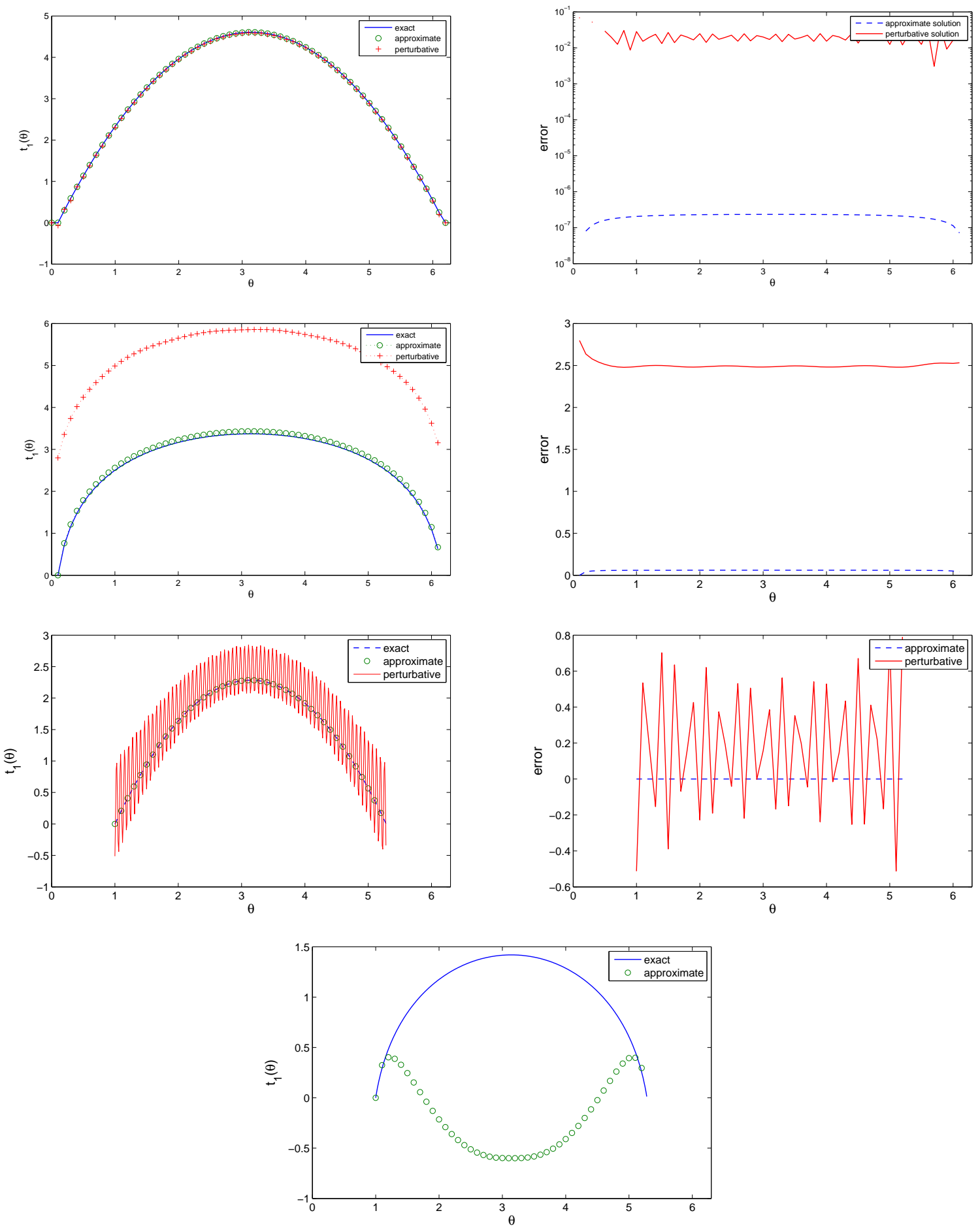

FIG. 2: Comparison between three approaches for computing $t_{1}(\theta)$ in 2D: the exact solution (20, 25), the approximation (32) and the perturbative formula (A15), with $D_{2}=1, a=0.01$. In the first row, the other parameters are: $\epsilon=0.1, \lambda=1$, and the series are truncated to $N=100$. On the right, the absolute error between the exact solution and the approximation (dashed blue curve) and between the exact solution and the perturbative formula (solid red curve). The approximation is very accurate indeed. In the second row, the parameters are: $\epsilon=0.1, \lambda=1000$, and the series are truncated to $N=100$ for the exact and approximate solutions, and to $N=1000$ for the perturbative solution. One can see that the perturbative solution is inaccurate for large values of $\lambda$, while the maximal relative error of the approximate solution is below $2 \%$. In the third row, the parameters are: $\epsilon=1, \lambda=1$, and the series are truncated to $N=100$. The perturbative solution is evidently not applicable. In the last row, the parameters are: $\epsilon=1, \lambda=1000$, and the series are truncated to $N=100$. In this case, the approximate solution significantly deviates from the exact one (providing mostly negative values). The perturbative solution is completely invalid (not shown). 

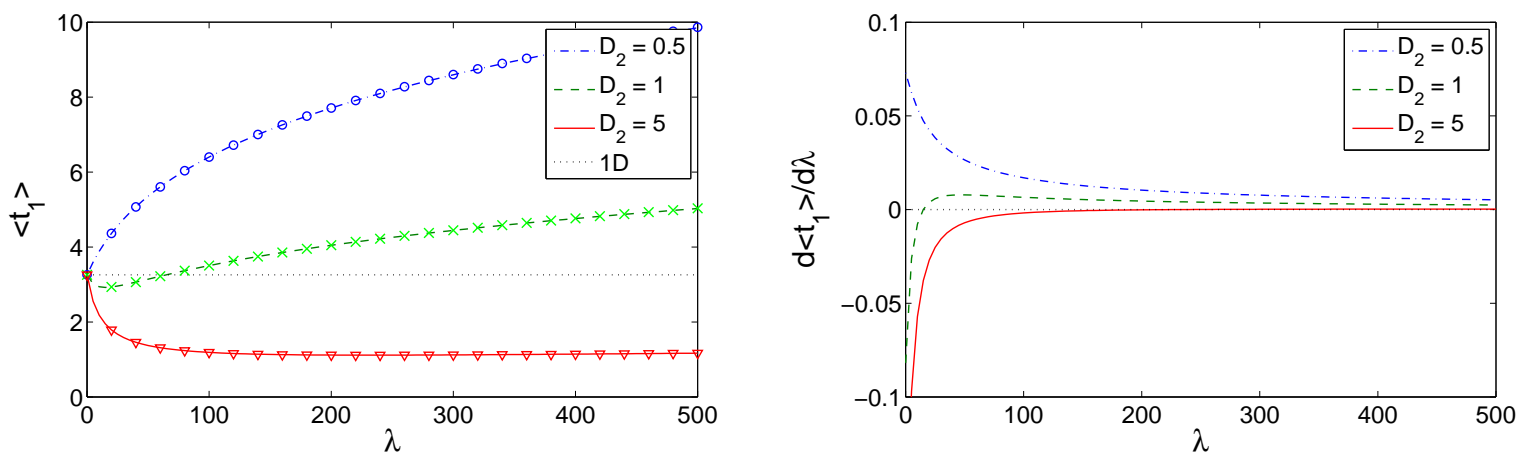

FIG. 3: Left: In 2D, the mean time $\left\langle t_{1}\right\rangle$ computed through Eq. 25, 29) with $N=100$ as a function of the desorption rate $\lambda$ for three values of $D_{2}: D_{2}=0.5$ (dot-dashed blue line), $D_{2}=1$ (dashed green line), and $D_{2}=5$ (solid red line). The other parameters are: $a=0.1$ and $\epsilon=0.01$. When $D_{2}<D_{2, \text { crit }} \approx 0.6348 \ldots$ (the first case), $\left\langle t_{1}\right\rangle$ monotonously increases with $\lambda$ so that there is no optimal value. In two other cases, $D_{2}>D_{2}$,crit, and $\left\langle t_{1}\right\rangle$ starts first to decrease with $\lambda$, passes through a minimum (the optimal value) and monotonously increases. Symbols show the approximate mean time computed through Eq. (31, 29). One can see that the approximation accurate enough even for large values of $\lambda$. Right: The derivative $\frac{d\left\langle t_{1}\right\rangle}{d \lambda}$ defined by Eq. 35 for the same parameters.
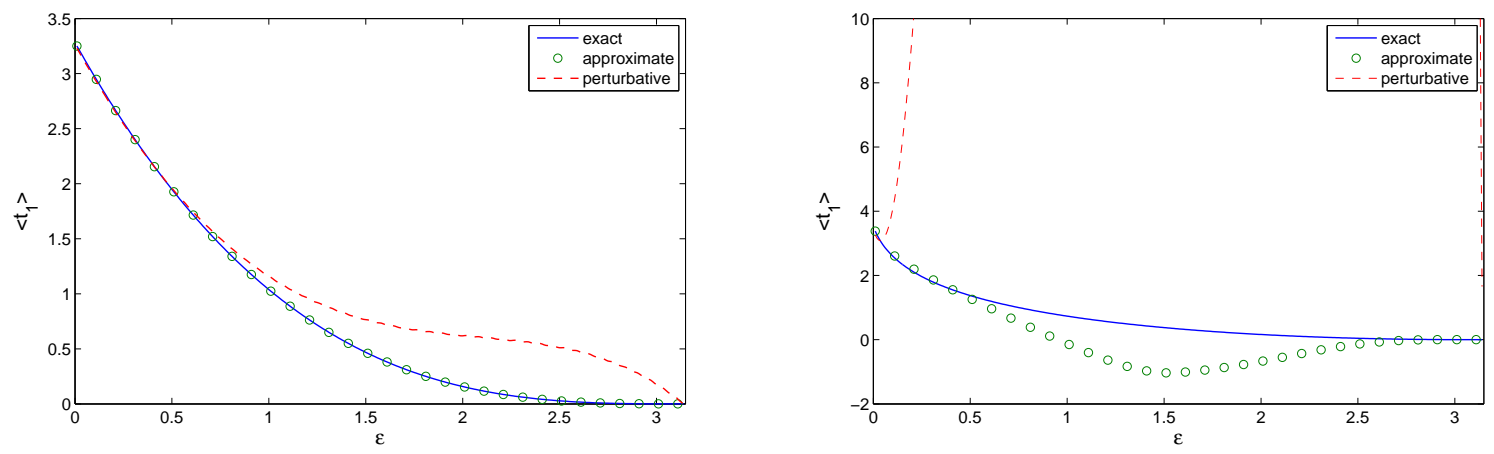

FIG. 4: In 2D, the mean time $\left\langle t_{1}\right\rangle$ as a function of $\epsilon$, with $D_{2}=1, a=0.01$, and $\lambda=1$ (left) or $\lambda=1000$ (right). The exact computation through Eq. (25, 29) is compared to the approximation (31, 29) and to the perturbative approach. In all cases, the series are truncated to $N=100$. For small $\lambda(\lambda=1)$, the approximate solution is very close to the exact one, while the perturbative solution is relatively close for $\epsilon$ up to 1 . In turn, for large $\lambda(\lambda=1000)$, the approximate solution shows significant deviations for the intermediate values of $\epsilon$, while the perturbative solution is not applicable at all.

where $\tilde{Q}=-Q \frac{R^{2}}{\pi D_{1}}, \eta=\frac{2 a R-a^{2}}{4 D_{2}}$. The derivative of $\left\langle t_{1}\right\rangle$ with respect to $\lambda$ is then

$$
\frac{\partial\left\langle t_{1}\right\rangle}{\partial \lambda}=\frac{R^{4} \eta}{2 \pi^{2} D_{1}^{2}}\left[\frac{2 \pi D_{1}}{3 R^{2}}(\pi-\epsilon)^{3}-\left(\xi \cdot \frac{\left(\eta^{-1}+2 \lambda\right) I+\lambda^{2} \tilde{Q}}{(I+\lambda \tilde{Q})^{2}} U\right)\right] .
$$

If the derivative is negative at $\lambda=0$, i.e.

$$
\eta \frac{2 \pi D_{1}}{3 R^{2}}(\pi-\epsilon)^{3}<(\xi \cdot U)
$$

bulk excursions are beneficial to the search. This inequality determines the critical value for the bulk diffusion coefficient $D_{2, \text { crit }}$ (which enters through $\eta$ ), above which bulk excursions are beneficial:

$$
\frac{D_{1}}{D_{2, \text { crit }}}=\frac{6 R^{2}(\xi \cdot U)}{\pi(\pi-\epsilon)^{3}\left(2 a R-a^{2}\right)}=\frac{24}{\pi(\pi-\epsilon)^{3}\left(1-x^{2}\right)} \sum_{n=1}^{\infty} \frac{1-x^{n}}{n^{4}}\left[(\pi-\epsilon) \cos (n \epsilon)+\frac{\sin (n \epsilon)}{n}\right]^{2} .
$$

Two comments are in order: 


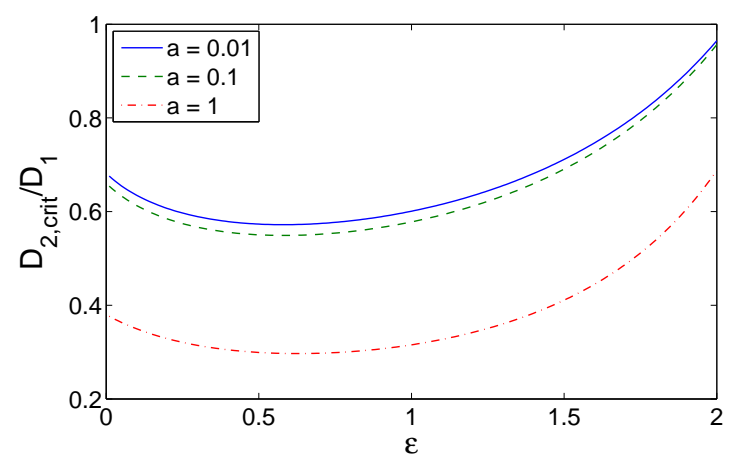

FIG. 5: $D_{2, \text { crit }}$ as a function of $\epsilon$ is computed from Eq. (37) in 2D for three values of $a / R$ : 0.01, 0.1, and 1. When $\epsilon$ approaches

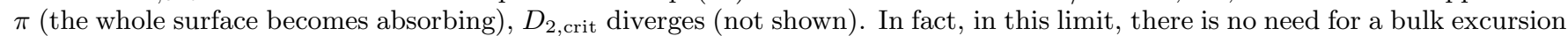
because the target will be found immediately by the surface diffusion.

(i) Interestingly, this ratio depends only on $a / R$ and $\epsilon$. In the limit of $\epsilon \rightarrow 0$, one gets

$$
\frac{D_{1}}{D_{2, \text { crit }}} \approx \frac{24}{\pi^{2}\left(1-x^{2}\right)} \sum_{n=1}^{\infty} \frac{1-x^{n}}{n^{4}} .
$$

Taking next the limit $a / R \rightarrow 0$ finally yields:

$$
\frac{D_{1}}{D_{2, \text { crit }}} \approx \frac{12 \zeta(3)}{\pi^{2}} \approx 1.4615
$$

where $\zeta$ stands for the Riemann $\zeta$-function.

(ii) The dependence of the rhs of Eq.(37) with $\epsilon$ is not trivial (Fig. 5). Indeed it can be proved to have a maximum with respect to $\epsilon$, which can be understood intuitively as follows: in the vicinity of $\epsilon=0$, increasing $\epsilon$ makes the constraint less stringent since the target can be reached directly from the bulk; in the opposite limit $\epsilon \rightarrow \pi$, the constraint on $D_{1} / D_{2}$ has to tend to 0 since the target is found immediately from the surface. Quantitatively, in the physical limit $a \rightarrow 0$, one finds that, as soon as $D_{2} / D_{1}>\left(D_{2, \text { crit }} / D_{1}\right) \approx 0.68 \ldots$, bulk excursions can be beneficial.

\section{When is there an optimal value of the desorption rate $\lambda$ minimizing the search time?}

If the reaction time $\left\langle t_{1}\right\rangle$ is a decreasing function of the desorption rate $\lambda$, the bulk excursions are "too favorable", and the best search strategy is obtained for $\lambda \rightarrow \infty$ (purely bulk search). For the reaction time to be an optimizable function of $\lambda$, the derivative $\frac{d\left\langle t_{1}\right\rangle}{d \lambda}$ has to be positive at some $\lambda$. This necessary and sufficient condition remains formal and requires numerical analysis of Eq. (35). A simple sufficient condition can be used instead by demanding that the search time at zero desorption rate is less than the search time at infinite desorption rate:

$$
\left\langle t_{1}(\lambda=0)\right\rangle<\left\langle t_{1}(\lambda \rightarrow \infty)\right\rangle .
$$

This writes in the physically relevant limit $a \ll R$ (using the result of [30]):

$$
\frac{D_{1}}{D_{2}}>\frac{(\pi-\epsilon)^{3}}{3 \pi c(\epsilon)}, \text { with } c(\epsilon) \equiv \frac{1}{\pi \sqrt{2}} \int_{0}^{\pi-\epsilon} \frac{u \sin (u / 2)}{\sqrt{\cos (u)+\cos (\epsilon)}} \mathrm{d} u
$$

Finally, combining Eqs. (37, 41), the search time is found to be an optimizable function of $\lambda$ in the limit $a \ll R$ if

$$
\frac{(\pi-\epsilon)^{3}}{3 \pi c(\epsilon)}<\frac{D_{1}}{D_{2}}<\frac{12}{\pi(\pi-\epsilon)^{3}} \sum_{n=1}^{\infty} \frac{1}{n^{3}}\left[(\pi-\epsilon) \cos (n \epsilon)+\frac{\sin (n \epsilon)}{n}\right]^{2} .
$$


Knowing that $c(\epsilon)=\ln (2 / \epsilon)+\mathcal{O}(\epsilon)$, Eq. (42) writes in the small $\epsilon$ limit:

$$
\frac{\pi^{2}}{3 \ln (2 / \epsilon)}<\frac{D_{1}}{D_{2}}<\frac{12 \zeta(3)}{\pi^{2}}
$$

which summarizes the conditions for the search time to be an optimizable function of $\lambda$. This case is illustrated in Fig. 10.

\section{3D CASE}

In this section, the confining domain is a sphere of radius $R$ and the target is the region on the boundary defined by $\theta \in[0, \epsilon]$, where $\theta$ is the elevation angle.

\section{A. Basic equations}

The 3D analogs of Eqs. (1, 2) read as

$$
\begin{aligned}
\frac{D_{1}}{R^{2}}\left(\frac{\partial^{2} t_{1}}{\partial \theta^{2}}+\frac{1}{\tan \theta} \frac{\partial t_{1}}{\partial \theta}\right)+\lambda\left[t_{2}(R-a, \theta)-t_{1}(\theta)\right] & =-1 \text { for } \theta \in[\epsilon, \pi], \\
D_{2}\left(\frac{\partial^{2}}{\partial r^{2}}+\frac{2}{r} \frac{\partial}{\partial r}+\frac{1}{r^{2}} \frac{\partial^{2}}{\partial \theta^{2}}\right) t_{2}(r, \theta) & =-1 .
\end{aligned}
$$

These equations have to be completed by two boundary conditions:

$$
\begin{aligned}
t_{2}(R, \theta) & =t_{1}(\theta), \\
t_{1}(\theta) & =0 \text { for } \theta \in[0, \epsilon],
\end{aligned}
$$

which respectively describe the adsorption events and express that the target is an absorbing region of the sphere.

\section{B. Integral equation for $t_{1}$}

One can search for a solution in the following form

$$
t_{2}(r, \theta)=\alpha_{0}-\frac{r^{2}}{6 D_{2}}+\sum_{n=1}^{\infty} \alpha_{n} r^{n} P_{n}(\cos \theta)
$$

where $P_{n}$ stands for the Legendre polynomial of order $n$. Using the orthonormality of Legendre polynomials, the projection of $t_{2}(R, \theta)$ on $P_{m}$ writes

$$
\int_{0}^{\pi} \sin \theta P_{m}(\cos \theta) t_{2}(R, \theta) \mathrm{d} \theta=2\left(\alpha_{0}-\frac{R^{2}}{6 D_{2}}\right) \delta_{m, 0}+\frac{2 \alpha_{m} R^{m}}{2 m+1} .
$$

Knowing that

$$
t_{2}(R, \theta)= \begin{cases}t_{1}(\theta) & \text { if } \theta \in[\epsilon, \pi], \\ 0 & \text { if } \theta \in[0, \epsilon]\end{cases}
$$

the $\alpha_{n}$ can be written in terms of $t_{1}(\theta)$ as

$$
\begin{aligned}
\alpha_{0}-\frac{R^{2}}{6 D_{2}} & =\frac{1}{2} \int_{\epsilon}^{\pi} \sin \theta t_{1}(\theta) \mathrm{d} \theta, \\
\alpha_{n} R^{n} & =\frac{2 n+1}{2} \int_{\epsilon}^{\pi} \sin \theta P_{n}(\cos \theta) t_{1}(\theta) \mathrm{d} \theta \text { if } n \geq 1 .
\end{aligned}
$$


Taylor expanding the rhs of

$$
\frac{\partial^{2} t_{1}}{\partial \theta^{2}}+\frac{1}{\tan \theta} \frac{\partial t_{1}}{\partial \theta}=-\frac{R^{2}}{D_{1}}-\omega^{2}\left[t_{2}(R-a, \theta)-t_{2}(R, \theta)\right]
$$

leads to

$$
\frac{\partial^{2} t_{1}}{\partial \theta^{2}}+\frac{1}{\tan \theta} \frac{\partial t_{1}}{\partial \theta}=-\frac{R^{2}}{D_{1}}-\omega^{2} \sum_{k=1}^{\infty} \frac{(-a)^{k}}{k !}\left(\frac{\partial^{k} t_{2}}{\partial r^{k}}\right)_{R, \theta}
$$

Using Eq. (48) for $t_{2}$ yields

$$
\frac{\partial^{2} t_{1}}{\partial \theta^{2}}+\frac{1}{\tan \theta} \frac{\partial t_{1}}{\partial \theta}=-\frac{R^{2}}{D_{1}}-\omega^{2}\left(\frac{a R}{3 D_{2}}-\frac{a^{2}}{6 D_{2}}\right)-\omega^{2} \sum_{k=1}^{\infty} \frac{(-a)^{k}}{k !} \sum_{n=k}^{\infty} \alpha_{n} n(n-1) \ldots(n-k+1) R^{n-k} P_{n}(\cos \theta) .
$$

Changing the order of summations over $n$ and $k$ and using the binomial formula and Eq. (51) for $\alpha_{n}$ finally give

$$
\frac{\partial^{2} t_{1}}{\partial \theta^{2}}+\frac{1}{\tan \theta} \frac{\partial t_{1}}{\partial \theta}=-\frac{R^{2}}{D_{1}}-\omega^{2}\left(\frac{a R}{3 D_{2}}-\frac{a^{2}}{6 D_{2}}\right)-\frac{\omega^{2}}{2} \sum_{n=1}^{\infty}\left(x^{n}-1\right) P_{n}(\cos \theta)(2 n+1) \int_{\epsilon}^{\pi} \sin \theta^{\prime} P_{n}\left(\cos \theta^{\prime}\right) t_{1}\left(\theta^{\prime}\right) \mathrm{d} \theta^{\prime}
$$

where, as in previous section, $x \equiv 1-\frac{a}{R}$. This integro-differential equation for $t_{1}$ can actually easily be transformed into an integral equation for $t_{1}$, by integrating successively two times. Indeed, multiplying first both members of Eq. (55) by $\sin \theta$ and integrating between $\pi$ and $\theta$ gives

$$
\begin{aligned}
\sin \theta t_{1}^{\prime}(\theta) & =\left[\frac{R^{2}}{D_{1}}+\omega^{2}\left(\frac{a R}{3 D_{2}}-\frac{a^{2}}{6 D_{2}}\right)\right](\cos \theta+1) \\
& +\frac{\omega^{2}}{2} \sum_{n=1}^{\infty}\left(x^{n}-1\right)\left(P_{n+1}(\cos \theta)-P_{n-1}(\cos \theta)\right) \int_{\epsilon}^{\pi} \sin \theta^{\prime} P_{n}\left(\cos \theta^{\prime}\right) t_{1}\left(\theta^{\prime}\right) \mathrm{d} \theta^{\prime},
\end{aligned}
$$

where we have used

$$
\int P_{n}(x) \mathrm{d} x=-\frac{1}{n(n+1)}\left(1-x^{2}\right) P_{n}^{\prime}(x)=\frac{1}{2 n+1}\left(P_{n+1}(x)-P_{n-1}(x)\right) .
$$

Dividing Eq. (56) by $\sin \theta$ and integrating between $\epsilon$ and $\theta$ finally leads to

$$
\begin{aligned}
t_{1}(\theta) & =2\left(\frac{R^{2}}{D_{1}}+\omega^{2}\left(\frac{a R}{3 D_{2}}-\frac{a^{2}}{6 D_{2}}\right)\right) \ln \left(\frac{\sin (\theta / 2)}{\sin (\epsilon / 2)}\right) \\
& +\frac{\omega^{2}}{2} \sum_{n=1}^{\infty}\left(x^{n}-1\right) \frac{2 n+1}{n(n+1)}\left(P_{n}(\cos \theta)-P_{n}(\cos \epsilon)\right) \int_{\epsilon}^{\pi} \sin \theta^{\prime} P_{n}\left(\cos \theta^{\prime}\right) t_{1}\left(\theta^{\prime}\right) \mathrm{d} \theta^{\prime},
\end{aligned}
$$

where we have again used Eq. (57), or equivalently to

$$
\psi(\theta)=\ln \left(\frac{1-\cos \theta}{1-\cos \epsilon}\right)+\Omega \sum_{n=1}^{\infty}\left(x^{n}-1\right) \frac{2 n+1}{n(n+1)}\left(P_{n}(\cos \theta)-P_{n}(\cos \epsilon)\right) \int_{\epsilon}^{\pi} \sin \left(\theta^{\prime}\right) P_{n}\left(\cos \theta^{\prime}\right) \psi\left(\theta^{\prime}\right) \mathrm{d} \theta^{\prime}
$$

with the following definitions

$$
\psi(\theta) \equiv \frac{t_{1}(\theta)}{\omega^{2} T}, \quad T \equiv \frac{1}{\lambda}+\frac{R^{2}-(R-a)^{2}}{6 D_{2}}, \quad \Omega \equiv \frac{\omega^{2}}{2}
$$

in this $3 \mathrm{D}$ case.

\section{Exact solution}

Iterating the integral equation Eq. (58) shows that the solution $\psi(\theta)$ writes for $\theta \in[\epsilon, \pi]$ :

$$
\psi(\theta)=\ln \left(\frac{1-\cos \theta}{1-\cos \epsilon}\right)+\sum_{n=1}^{\infty} d_{n}\left[P_{n}(\cos \theta)-P_{n}(\cos \epsilon)\right]
$$


with the coefficients $d_{n}$ which satisfy

$$
\sum_{n=1}^{\infty} d_{n}\left[P_{n}(\cos \theta)-P_{n}(\cos \epsilon)\right]=\Omega \sum_{n=1}^{\infty}\left(U_{n}+\sum_{n^{\prime}=1}^{\infty} Q_{n, n^{\prime}} d_{n^{\prime}}\right)\left[P_{n}(\cos \theta)-P_{n}(\cos \epsilon)\right]
$$

where we introduced the new definitions

$$
\begin{aligned}
& U_{n} \equiv \frac{\left(x^{n}-1\right)(2 n+1)}{n(n+1)} \int_{\epsilon}^{\pi} \mathrm{d} \theta^{\prime} \sin \left(\theta^{\prime}\right) P_{n}\left(\cos \theta^{\prime}\right) \ln \left(\frac{1-\cos \theta^{\prime}}{1-\cos \epsilon}\right)=\frac{\left(1-x^{n}\right)(2 n+1)}{n^{2}(n+1)^{2}} \xi_{n}, \\
& \xi_{n} \equiv\left(1+\frac{n \cos \epsilon}{n+1}\right) P_{n}(\cos \epsilon)+\frac{P_{n-1}(\cos \epsilon)}{n+1} \quad(n=1,2, \ldots),
\end{aligned}
$$

and

$$
Q_{n, n^{\prime}} \equiv-\frac{\left(1-x^{n}\right)(2 n+1)}{n(n+1)} I_{\epsilon}\left(n, n^{\prime}\right) \quad\left(n, n^{\prime}=1,2, \ldots\right)
$$

with

$$
I_{\epsilon}\left(n, n^{\prime}\right) \equiv \int_{-1}^{\cos \epsilon} P_{n}(u)\left(P_{n^{\prime}}(u)-P_{n^{\prime}}(\cos \epsilon)\right) \mathrm{d} u
$$

In Appendix C, we compute this integral explicitly.

Since Eq. (58) should be satisfied for any $\theta$, one gets $\mathbf{d}=\Omega(U+Q \mathbf{d})$, from which

$$
d_{n}=\Omega\left[(I-\Omega Q)^{-1} U\right]_{n} \quad(n=1,2, \ldots) .
$$

As in 2D, using the series expansion of $(I-\Omega Q)^{-1}$, Eq. (65) can be seen as a series in powers of $\Omega$, whose $n$-th order coefficient can be explicitly written in terms of the $n$-th power of the matrix $Q$.

Note that the first term in Eq. (60) can also be represented as a series

$$
\sum_{n=1}^{\infty} e_{n}\left[P_{n}(\cos \theta)-P_{n}(\cos \epsilon)\right]=\left\{\begin{array}{cc}
\ln \left(\frac{1-\cos \theta}{1-\cos \epsilon}\right), & \epsilon<\theta<\pi-\epsilon \\
0, & \text { otherwise }
\end{array}\right.
$$

where the coefficients $e_{n}$ are obtained by multiplying this equation by $P_{n}(\cos \theta) \sin \theta$ and integrating from 0 to $\pi$ :

$$
e_{n}=-\frac{2 n+1}{2 n(n+1)} \xi_{n}
$$

Once the $d_{n}$ determined, the search time $\left\langle t_{1}\right\rangle$ can be written as

$$
\left\langle t_{1}\right\rangle \equiv \frac{\omega^{2} T}{2} \int_{\epsilon}^{\pi} d \theta \sin \theta \psi(\theta)=\frac{\omega^{2} T}{2}\left\{2 \ln \left(\frac{2}{1-\cos \epsilon}\right)-(1+\cos \epsilon)-\sum_{n=1}^{\infty} d_{n} \xi_{n}\right\}
$$

\section{Perturbative solution}

The first terms of a perturbative expansion with respect to $\epsilon$ can easily be obtained from the previous exact solution. At leading order in $\epsilon$, we have

$$
\begin{gathered}
U_{n}=U_{n}^{(0)}+O(\epsilon)=\frac{2\left(1-x^{n}\right)(2 n+1)}{n^{2}(n+1)^{2}}+O(\epsilon), \\
Q_{m, n}=Q_{m, n}^{(0)}+O(\epsilon)=-\frac{2\left(1-x^{n}\right)}{n(n+1)} \delta_{m, n}+O(\epsilon),
\end{gathered}
$$

from which

$$
d_{n}=\Omega\left[\left(I-\Omega Q^{(0)}\right)^{-1} U^{(0)}\right]_{n}+O(\epsilon)=\frac{2 \Omega}{n(n+1)} \frac{\left(1-x^{n}\right)(2 n+1)}{n(n+1)+2 \Omega\left(1-x^{n}\right)}+O(\epsilon)
$$


One finds therefore

$$
\psi(\theta)=-2 \ln \epsilon+2 \ln (2 \sin (\theta / 2))-2 \Omega \sum_{n=1}^{\infty}\left(1-x^{n}\right) \frac{2 n+1}{n(n+1)} \frac{1-P_{n}(\cos \theta)}{n(n+1)+2 \Omega\left(1-x^{n}\right)}+O(\epsilon) .
$$

Averaging over $\theta$, it finally yields:

$$
\left\langle t_{1}\right\rangle \approx \omega^{2} T\left\{-2 \ln (\epsilon / 2)-1-2 \Omega \sum_{n=1}^{\infty} \frac{2 n+1}{n(n+1)} \frac{\left(1-x^{n}\right)}{n(n+1)+2 \Omega\left(1-x^{n}\right)}+O(\epsilon)\right\} .
$$

This result was given in [37] without derivation.

\section{E. Approximate solution}

As earlier for the 2D case, an approximate solution can be derived. As shown numerically (see Figs. 6, 7, 8 and section $\mathrm{V}$ for more details about numerical methods), this approximation of $t_{1}$ proves to be in quantitative agreement with the exact expression for a wide range of parameters.

This approximation relies on the fact that, in the small target size limit $\epsilon \rightarrow 0$, the matrix $Q$ is diagonal, which in turn mirrors the orthogonality of $\left\{P_{n}(\cos \theta)\right\}_{n}$ on $[0, \pi]$. More precisely, one has

$$
Q_{m, n}=\delta_{m, n} Q_{n, n}+\mathcal{O}\left(\epsilon^{4}\right)
$$

and keeping only the leading term of this expansion yields

$$
d_{n} \approx \Omega\left(1-\Omega Q_{n, n}\right)^{-1} U_{n}
$$

from which

$$
\psi(\theta) \approx \ln \left(\frac{1-\cos \theta}{1-\cos \epsilon}\right)+\Omega \sum_{n=1}^{\infty}\left(1-x^{n}\right) \frac{2 n+1}{n(n+1)}\left(P_{n}(\cos \theta)-P_{n}(\cos \epsilon)\right) \frac{\left(1+\frac{n \cos \epsilon}{n+1}\right) P_{n}(\cos \epsilon)+\frac{P_{n-1}(\cos \epsilon)}{n+1}}{n(n+1)+\Omega\left(1-x^{n}\right)(2 n+1) I_{\epsilon}(n, n)} .
$$

The mean time $\left\langle t_{1}\right\rangle$ is then approximated as

$$
\begin{aligned}
\left\langle t_{1}\right\rangle & \approx \omega^{2} T\left\{\ln \left(\frac{2}{1-\cos \epsilon}\right)-\frac{1+\cos \epsilon}{2}\right. \\
& \left.\left.-\frac{\Omega}{2} \sum_{n=1}^{\infty} \frac{\left(1-x^{n}\right)(2 n+1)}{n(n+1)} \frac{\left[\left(1+\frac{n \cos \epsilon}{n+1}\right) P_{n}(\cos \epsilon)+\frac{P_{n-1}(\cos \epsilon)}{n+1}\right]^{2}}{n(n+1)+\Omega\left(1-x^{n}\right)(2 n+1) I_{\epsilon}(n, n)}\right]\right\} .
\end{aligned}
$$

\section{F. Variations of the search time $\left\langle t_{1}\right\rangle$ with the desorption rate $\lambda$}

We investigate here as in the $2 \mathrm{D}$ case the dependence of $\left\langle t_{1}\right\rangle$ on $\lambda$.

\section{When are bulk excursions beneficial to the search?}

The sign of $\frac{\partial\left\langle t_{1}\right\rangle}{\partial \lambda}$ at $\lambda=0$ is conveniently studied by rewriting Eq. (68) as

$$
\left\langle t_{1}\right\rangle=\frac{R^{4}}{4 D_{1}^{2}}(1+\lambda \eta)\left\{\frac{4 D_{1}}{R^{2}}\left[\ln \left(\frac{2}{1-\cos \epsilon}\right)-\frac{1+\cos \epsilon}{2}\right]-\lambda\left(\xi \cdot(I+\lambda \tilde{Q})^{-1} U\right)\right\},
$$

where $\tilde{Q}=-Q \frac{R^{2}}{2 D_{1}}$ and $\eta=\frac{2 a R-a^{2}}{6 D_{2}}$. The derivative of $\left\langle t_{1}\right\rangle$ with respect to $\lambda$ is then

$$
\frac{\partial\left\langle t_{1}\right\rangle}{\partial \lambda}=\frac{R^{4} \eta}{4 D_{1}^{2}}\left\{\left[\ln \left(\frac{2}{1-\cos \epsilon}\right)-\frac{1+\cos \epsilon}{2}\right]-\left(\xi \cdot \frac{\left(\eta^{-1}+2 \lambda\right) I+\lambda^{2} \tilde{Q}}{(I+\lambda \tilde{Q})^{2}} U\right)\right\} .
$$



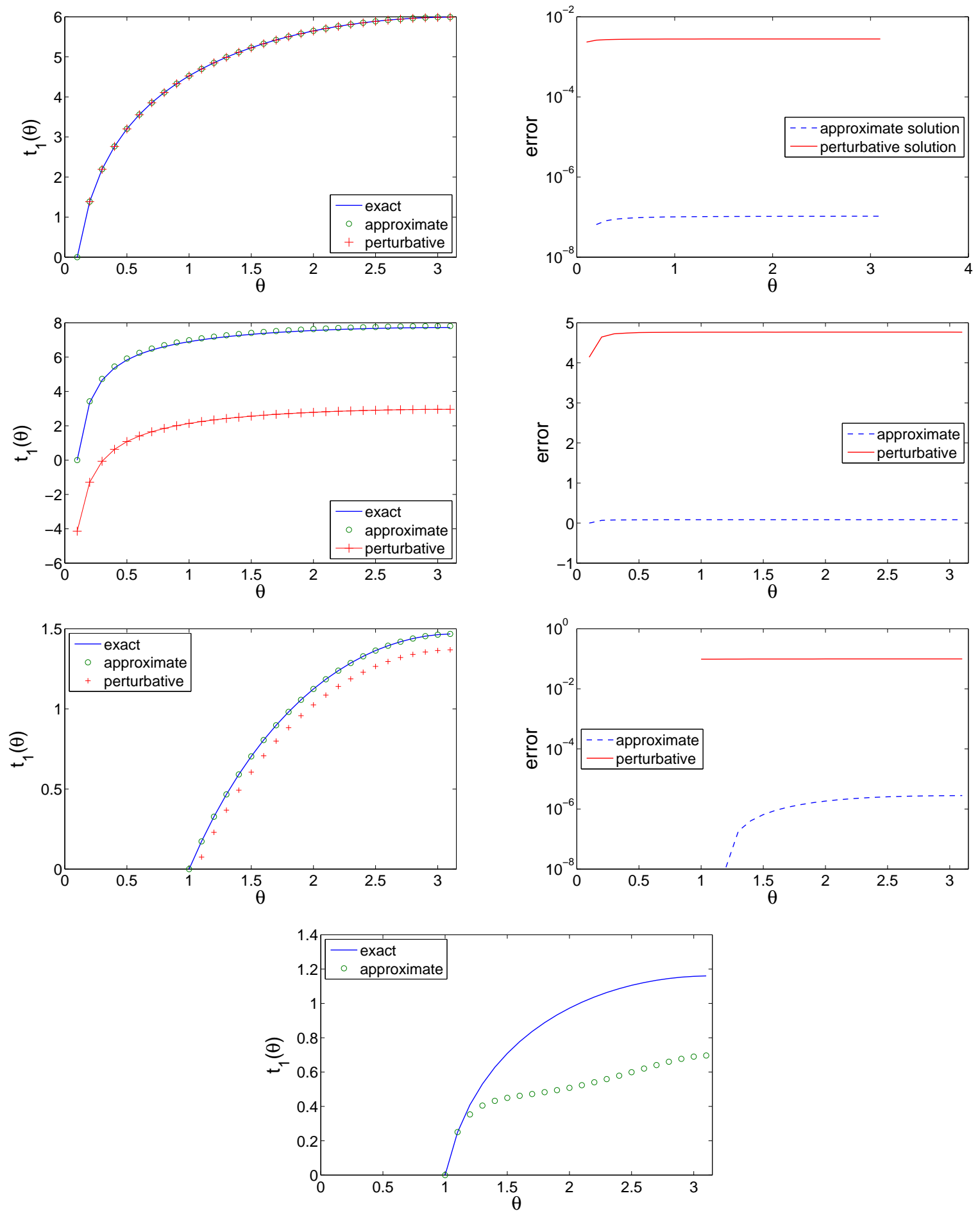

FIG. 6: Comparison between three approaches for computing $t_{1}(\theta)$ in 3D: the exact solution (60) 65), the approximation (75) and the perturbative formula (71), with $D_{2}=1, a=0.01$. In the first row, the other parameters are: $\epsilon=0.1, \lambda=1$, and the series are truncated to $N=100$. On the right, the absolute error between the exact solution and the approximation (dashed blue curve) and between the exact solution and the perturbative formula (solid red curve). The approximation is very accurate indeed. In the second row, the parameters are: $\epsilon=0.1, \lambda=1000$, and the series are truncated to $N=100$. One can see that the perturbative solution is inaccurate for large values of $\lambda$, while the maximal relative error of the approximate solution is still small. In the third row, the parameters are: $\epsilon=1, \lambda=1$, and the series are truncated to $N=100$. The perturbative solution is inaccurate as expected for large $\epsilon$. In the last row, the parameters are: $\epsilon=1, \lambda=1000$, and the series are truncated to $N=100$. In this case, the approximate solution deviates from the exact one for. The perturbative solution is negative and not shown. 

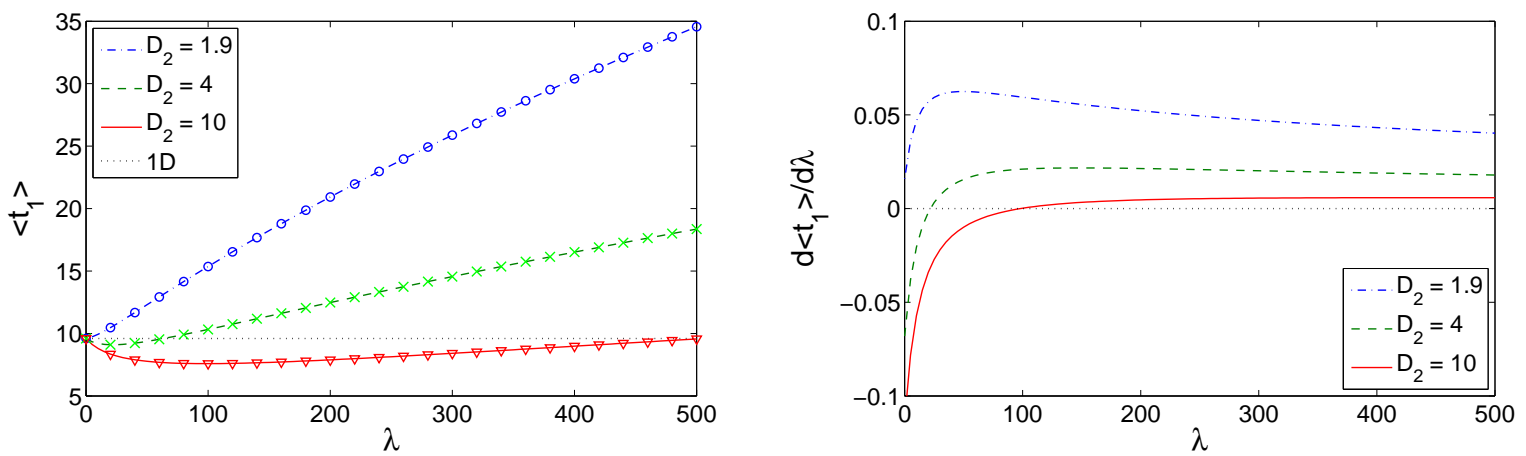

FIG. 7: Left: In 3D, the mean time $\left\langle t_{1}\right\rangle$ computed through Eq. 65, 68) with $N=100$ as a function of the desorption rate $\lambda$ for three values of $D_{2}: D_{2}=1.9$ (dot-dashed blue line), $D_{2}=4$ (dashed green line), and $D_{2}=10$ (solid red line). The other parameters are: $a=0.1$ and $\epsilon=0.01$. When $D_{2}<D_{2, \text { crit }} \approx 1.9997 \ldots$ (the first case), $\left\langle t_{1}\right\rangle$ monotonously increases with $\lambda$ so that there is no optimal value. In two other cases, $D_{2}>D_{2}$,crit, and $\left\langle t_{1}\right\rangle$ starts first to decrease with $\lambda$, passes through a minimum (the optimal value) and then increases. Symbols show the approximate mean time computed through Eq. (76). One can see that the approximation accurate enough even for large values of $\lambda$. Right: The derivative $\frac{d\left\langle t_{1}\right\rangle}{d \lambda}$ defined by Eq. (78) for the same parameters.
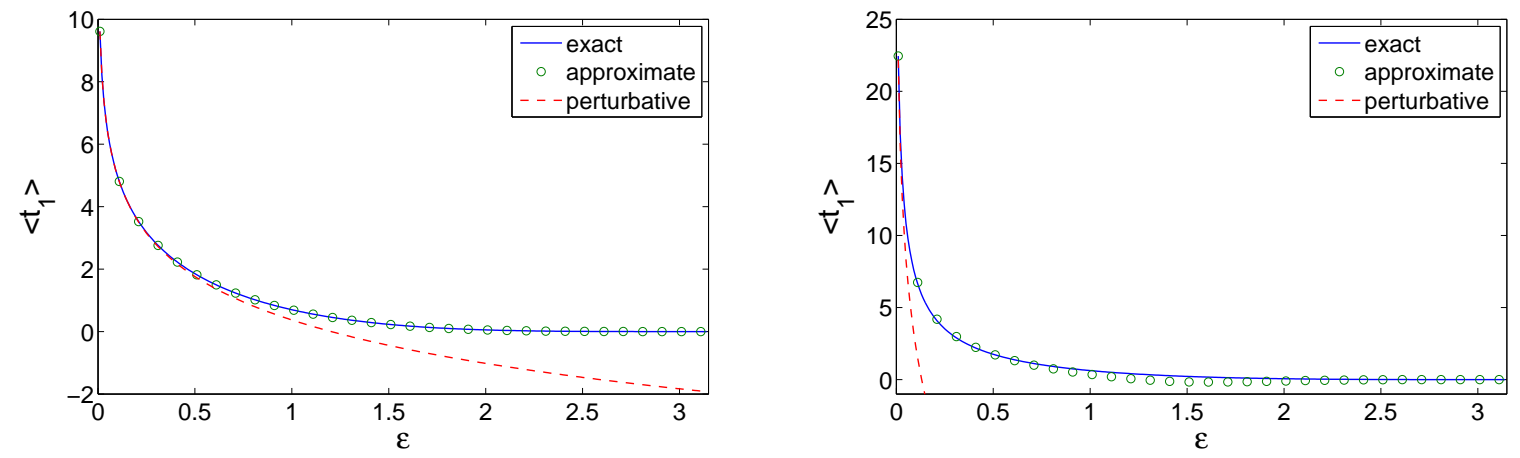

FIG. 8: In 3D, the mean time $\left\langle t_{1}\right\rangle$ as a function of $\epsilon$, with $D_{2}=1, a=0.01$, and $\lambda=1$ (left) or $\lambda=1000$ (right). The exact computation through Eq. (65) 68) is compared to the approximation (76) and to the perturbative approach. In all cases, the series are truncated to $N=100$. For small $\lambda(\lambda=1)$, the approximate solution is very close to the exact one, while the perturbative solution is relatively close for $\epsilon$ up to 1 . In turn, for large $\lambda(\lambda=1000)$, the approximate solution shows significant deviations for the intermediate values of $\epsilon$, while the perturbative solution is not applicable at all.

If the above derivative is negative at $\lambda=0$, i.e.

$$
\frac{4 D_{1}}{R^{2}}\left(\ln \left(\frac{2}{1-\cos \epsilon}\right)-\frac{1+\cos \epsilon}{2}\right)<\frac{(\xi \cdot U)}{\eta}
$$

bulk excursions are beneficial to the search. This inequality determines the critical value for the bulk diffusion coefficient $D_{2, \text { crit }}$ (which enters through $\eta$ ):

$$
\begin{aligned}
\frac{D_{2, \text { crit }}}{D_{1}} & =\left(\ln \left(\frac{2}{1-\cos \epsilon}\right)-\frac{1+\cos \epsilon}{2}\right) \frac{2\left(2 a R-a^{2}\right)}{3 R^{2}(\xi \cdot U)} \\
& =\left(\ln \left(\frac{2}{1-\cos \epsilon}\right)-\frac{1+\cos \epsilon}{2}\right) \frac{2\left(1-x^{2}\right)}{3} \\
& \times\left(\sum_{n=1}^{\infty} \frac{\left(1-x^{n}\right)(2 n+1)}{n^{2}(n+1)^{4}}\left[(n+1+n \cos \epsilon) P_{n}(\cos \epsilon)+P_{n-1}(\cos \epsilon)\right]^{2}\right)^{-1} .
\end{aligned}
$$




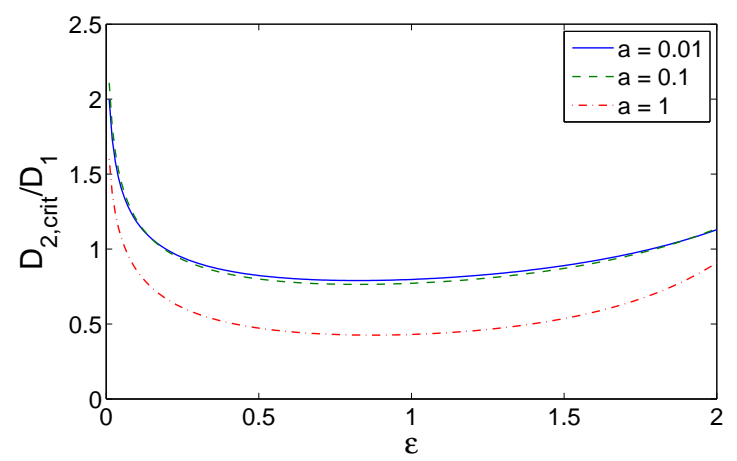

FIG. 9: $D_{2, \text { crit }}$ as a function of $\epsilon$ is computed from Eq. (80) in 3D for three values of $a / R: 0.01,0.1$, and 1 . When $\epsilon$ approaches

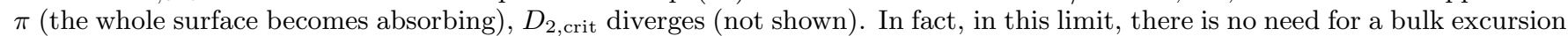
because the target will be found immediately by the surface diffusion. In addition, $D_{2, \text { crit }}$ also diverges as $\epsilon \rightarrow 0$ because a point-like target cannot be detected neither by bulk excursions, nor by surface diffusion in 3D.

In the limit of $\epsilon \rightarrow 0$, one gets

$$
\frac{D_{2, \text { crit }}}{D_{1}} \approx \frac{(2 \ln (2 / \epsilon)-1)\left(1-x^{2}\right)}{6}\left(\sum_{n=1}^{\infty} \frac{\left(1-x^{n}\right)(2 n+1)}{n^{2}(n+1)^{2}}\right)^{-1}+O(\epsilon) .
$$

In the physically relevant limit $a \ll R$, one has

$$
\frac{D_{2, \mathrm{crit}}}{D_{1}} \approx \frac{(2 \ln (2 / \epsilon)-1)}{3}\left(\sum_{n=1}^{\infty} \frac{2 n+1}{n(n+1)^{2}}\right)^{-1}+O(\epsilon)=\frac{2(2 \ln (2 / \epsilon)-1)}{\pi^{2}}+O(\epsilon) .
$$

There are similarities and differences between the behaviors of $D_{2, \text { crit }}$ in 2D and 3D. Figure 9 shows that $D_{2, \text { crit }}$ from Eq. (82) is not a monotonous function of $\epsilon$, with the qualitative explanation which is the same as in the two-dimensional case.

In contrast to the analogous Eq. (39) in 2D, the rhs of Eq. (82) diverges as $\epsilon \rightarrow 0$. This divergence reflects the fact that a poink-like target $(\epsilon=0)$, which could be found within a finite time in 2D by one-dimensional surface diffusion on the circle, is not detectable in 3D neither by bulk excursions, nor by surface diffusion.

\section{When is there an optimal value of the desorption rate $\lambda$ minimizing the search time?}

For the reaction time to be an optimizable function of the desorption rate $\lambda$, it is necessary to write an additional condition, requiring that the bulk excursions are not "too favorable" (otherwise, the best strategy is obtained for $\lambda \rightarrow \infty)$. A sufficient condition is given by demanding that the search time at zero desorption rate (i.e., without leaving the boundary) is less than the search time at infinite desorption rate

$$
\begin{gathered}
\left\langle t_{1}(\lambda=0)\right\rangle\left\langle\left\langle t_{1}(\lambda \rightarrow \infty)\right\rangle .\right. \\
\left\langle t_{1}(\lambda=0)\right\rangle=\frac{R^{2}}{D_{1}}(2 \ln (2 / \epsilon)-1)+O(\epsilon),
\end{gathered}
$$

which writes in the physically relevant limit $a \ll R$ (using the result of [38]):

$$
\left\langle t_{1}(\lambda \rightarrow \infty)\right\rangle=\frac{\pi R^{2}}{3 \epsilon D_{2}}(1+\epsilon \ln (1 / \epsilon)) .
$$

Finally, this conditions leads, for small $\epsilon$, to

$$
\frac{D_{1}}{D_{2}}>\frac{3 \epsilon}{\pi}(2 \ln (2 / \epsilon)-1)
$$



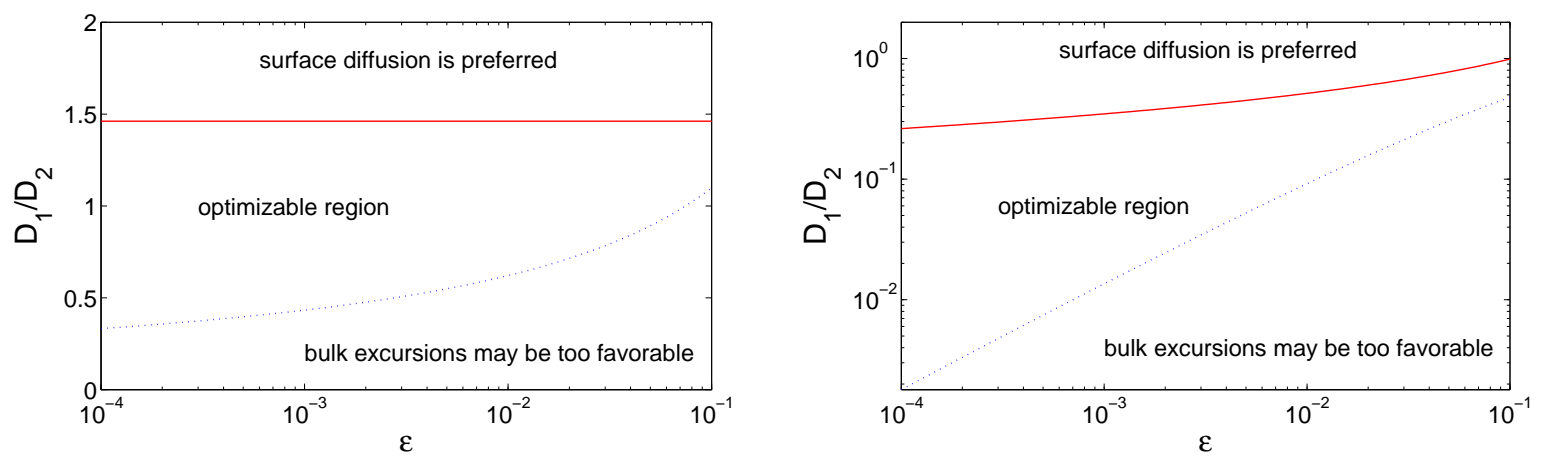

FIG. 10: The regions of optimality for the search time in 2D (left) and 3D (right). The lower bound (solid blue line) and upper bound (dashed red line) are given in the limit of $a \ll R$ and $\epsilon \ll 1$ by Eqs. (43, 87) in 2D and 3D, respectively. When the ratio $D_{1} / D_{2}$ lies between two curves, the search time $\left\langle t_{1}\right\rangle$ is optimizable with respect to $\lambda$. Above the upper bound, surface diffusion is preferred $(\lambda=0$ is the optimal solution), while below the lower bound, bulk excursions may be "too favorable" ( $\lambda \rightarrow \infty$ may give the optimal solution). We recall that the lower bound was obtained from the sufficient condition (83) meaning that the region below the dotted line may still be optimizable.

Combining the two conditions (82, 86), the search time is found to be optimizable when $a \ll R$ and $\epsilon \ll 1$ if

$$
\frac{3 \epsilon}{\pi}(2 \ln (2 / \epsilon)-1)<\frac{D_{1}}{D_{2}}<\frac{\pi^{2}}{2(2 \ln (2 / \epsilon)-1)} .
$$

\section{NUMERICAL RESOLUTION}

In the previous sections, we derived the closed matrix forms (25) 65) for the coefficients $d_{n}$ in 2D and 3D. These coefficients determine the angular dependence of $t_{1}(\theta)$ through the explicit representations (20, 60) in 2D and 3D, respectively. Although the formulas (25, 65) which are based on the inversion of an infinite-dimensional matrix $(I-\Omega Q)$ remain implicit, a numerical resolution of the problem has become straightforward. In fact, one needs to truncate the infinite-dimensional matrix $Q$ and vectors $U$ and $\tilde{U}$ and to invert the truncated matrix $(I-\Omega Q)$ numerically.

There are six parameters that determine the function $t_{1}(\theta)$ : the radius $R$ of the disk (sphere), the diffusion coefficients $D_{1}$ and $D_{2}$, the desorption rate $\lambda$, the size $\epsilon$ of the absorbing region, and the distance $a$. From now on, we set the units of length and time by setting $R=1$ and $D_{1}=1$. Although the distance $a$ may take any value from 0 to $R$, the physically interesting case corresponds to the limit of small $a$. As we mentioned previously, the limit $a=0$ exists but trivially leads to searching on the surface, without intermediate bulk excursions. In order to reveal the role of $a$, we consider several values of $a$ : $0.001,0.01,0.1$ and 1 , the latter corresponding to the specific situation when search is always restarted from the center. Since the diffusion coefficient $D_{2}$ enters only through the prefactor $T$ from Eq. (10), its influence onto the searching time $t_{1}$ is easy to examine. In what follows, we take three values of $D_{2}: 0.1$, 1 and 10. The dependence of $t_{1}$ on the desorption rate $\lambda$ and the size $\epsilon$ is the most interesting issue which will be studied below.

In the previous sections, we derived several formulas for computing $t_{1}$ :

- explicit representations (20, 60) with the exact expressions (25, 65) for the coefficients;

- approximations (32, 75) which were derived by neglecting non-diagonal elements of the matrix $Q$;

- perturbative formulas (11, 71) which are valid for small $\epsilon$.

For a numerical computation of the coefficients in Eqs. (25, 65), we truncate the infinite-dimensional matrix $Q$ to a finite size $N \times N$ and invert the matrix $(I-\Omega Q)$. In order to check the accuracy of this scheme, we compute the coefficients by taking several values of $N$ from 10 to 200. For $D_{2}=1, \epsilon=0.1, a=0.01$ and $\lambda=1$, the computed mean time $\left\langle t_{1}\right\rangle$ rapidly converges to a limit. Even the computation with $N=10$ gives the result with four significant digits. Note that other sets of parameters (e.g., larger values of $\Omega$ ) may require larger truncation sizes. 


\section{CONCLUSION}

To conclude, we have presented an exact calculation of the mean first-passage time to a target on the surface of a $2 \mathrm{D}$ and 3D spherical domain, for a molecule performing surface-mediated diffusion. The presented approach is based on an integral equation which can be solved analytically, and numerically validated approximation schemes, which provide more tractable expressions of the mean FPT. This minimal model of surface-mediated reactions, which explicitly takes into account the combination of surface and bulk diffusions, shows the importance of correlations induced by the coupling of the switching dynamics to the geometry of the confinement. Indeed, standard MF treatments prove to substantially underestimate the reaction time in this case [5], and sometimes even fail to reproduce the proper monotonicity [34]. In the context of interfacial systems in confinement, our results show that the reaction time can be minimized as a function of the desorption rate from the surface, which puts forward a general mechanism of enhancement and regulation of chemical reactivity.

\section{Appendix A: Another approach in 2D}

In this Appendix, we describe another theoretical approach which relies on the explicit form of the Green function of the Poisson equation in $2 \mathrm{D}$ case. In particular, the perturbative analysis for small $\epsilon$ becomes easier within this approach.

Considering $t_{2}$ as a source term in the Poisson type equation (1) with absorbing conditions at $\theta=\epsilon$ and $\theta=2 \pi-\epsilon$ whose Green function is well known [39], $t_{1}$ writes

$$
t_{1}(\theta)=\frac{1}{\omega \sinh (2 \omega(\pi-\epsilon))} \int_{\epsilon}^{2 \pi-\epsilon} \sinh \left(\omega\left(\theta_{<}-\epsilon\right)\right) \sinh \left(\omega\left(2 \pi-\epsilon-\theta_{>}\right)\right)\left[\frac{R^{2}}{D_{1}}+\frac{\lambda R^{2}}{D_{1}} t_{2}\left(R-a, \theta^{\prime}\right)\right] \mathrm{d} \theta^{\prime}
$$

and the notations $\theta_{<}=\min \left(\theta, \theta^{\prime}\right)$ and $\theta_{>}=\max \left(\theta, \theta^{\prime}\right)$.

Injecting Eq. (5) into Eq. (A1) leads to

$$
t_{1}(\theta)=\frac{\omega}{\lambda \sinh (2 \omega(\pi-\epsilon))}\left(\mathrm{I}(0, \theta)\left(1+\lambda\left(\alpha_{0}-\frac{(R-a)^{2}}{4 D_{2}}\right)\right)+\lambda \sum_{m=1}^{\infty} \alpha_{m}(R-a)^{m} \mathrm{I}(m, \theta)\right),
$$

where, for $m$ integer,

$$
\begin{aligned}
\mathrm{I}(m, \theta) & \equiv \int_{\epsilon}^{2 \pi-\epsilon} \sinh \left(\omega\left(\theta_{<}-\epsilon\right)\right) \sinh \left(\omega\left(2 \pi-\epsilon-\theta_{>}\right)\right) \cos \left(m \theta^{\prime}\right) \mathrm{d} \theta^{\prime} \\
& =\frac{\omega}{\omega^{2}+m^{2}}(\cos (m \theta) \sinh (2 \omega(\pi-\epsilon))-2 \cos (m \epsilon) \sinh (\omega(\pi-\epsilon)) \cosh (\omega(\theta-\pi))),
\end{aligned}
$$

so that

$$
\begin{aligned}
t_{1}(\theta) & =\frac{1}{\lambda}+\alpha_{0}-\frac{(R-a)^{2}}{4 D_{2}}+\omega^{2} \sum_{m=1}^{\infty} \frac{\alpha_{m}}{\omega^{2}+m^{2}}(R-a)^{m} \cos (m \theta) \\
& -\frac{\cosh (\omega(\theta-\pi))}{\cosh (\omega(\pi-\epsilon))}\left(\frac{1}{\lambda}+\alpha_{0}-\frac{(R-a)^{2}}{4 D_{2}}+\omega^{2} \sum_{m=1}^{\infty} \frac{\alpha_{m}}{\omega^{2}+m^{2}}(R-a)^{m} \cos (m \epsilon)\right) .
\end{aligned}
$$

Substituting Eq. (A4) into Eq. (7) gives

$$
S \frac{\tanh (\omega(\pi-\epsilon))}{\omega \pi}=-\frac{\epsilon}{\pi} \lambda \alpha_{0}+1-\frac{\epsilon}{\pi}+\lambda\left(\frac{R^{2}}{4 D_{2}}-\left(1-\frac{\epsilon}{\pi}\right) \frac{(R-a)^{2}}{4 D_{2}}\right)-\frac{\lambda \omega^{2}}{\pi} \sum_{m=1}^{\infty} \frac{\alpha_{m}}{\omega^{2}+m^{2}}(R-a)^{m} \frac{\sin (m \epsilon)}{m}
$$


and

$$
\begin{aligned}
& \lambda\left(R^{n}-\frac{\omega^{2}}{\omega^{2}+n^{2}}\left(1-\frac{\epsilon}{\pi}\right)(R-a)^{n}\right) \alpha_{n}= \\
- & \frac{2 \sin (n \epsilon)}{n \pi}\left(1+\lambda\left(\alpha_{0}-\frac{(R-a)^{2}}{4 D_{2}}\right)\right) \\
- & \frac{\lambda \omega^{2}}{\pi}\left(\sum_{m \neq n} \frac{\alpha_{m}}{\omega^{2}+m^{2}}(R-a)^{m} \frac{\sin ((m-n) \epsilon)}{m-n}+\sum_{m=1}^{\infty} \frac{\alpha_{m}}{\omega^{2}+m^{2}}(R-a)^{m} \frac{\sin ((m+n) \epsilon)}{m+n}\right) \\
- & \frac{2 \tanh (\omega(\pi-\epsilon))}{\pi\left(\omega^{2}+n^{2}\right)} S\left(\omega \cos (n \epsilon)-\frac{n \sin (n \epsilon)}{\tanh (\omega(\pi-\epsilon))}\right),
\end{aligned}
$$

where

$$
S \equiv 1+\lambda\left(\alpha_{0}-\frac{(R-a)^{2}}{4 D_{2}}\right)+\lambda \omega^{2} \sum_{m=1}^{\infty} \frac{\alpha_{m}}{\omega^{2}+m^{2}}(R-a)^{m} \cos (m \epsilon) .
$$

Eq. (A5) can be rearranged into

$$
\begin{aligned}
\left(\alpha_{0}-\frac{R^{2}}{4 D_{2}}\right)\left(\frac{\epsilon}{\pi}+\frac{\tanh (\omega(\pi-\epsilon))}{\pi \omega}\right) & =\left(\frac{1}{\lambda}+\left(\frac{R^{2}}{4 D_{2}}-\frac{(R-a)^{2}}{4 D_{2}}\right)\right)\left(1-\frac{\epsilon}{\pi}-\frac{\tanh (\omega(\pi-\epsilon))}{\pi \omega}\right) \\
& -\frac{\omega}{\pi} \sum_{n=1}^{\infty} \frac{(R-a)^{n}}{\omega^{2}+n^{2}}\left(\tanh (\omega(\pi-\epsilon)) \cos (n \epsilon)+\frac{\omega}{n} \sin (n \epsilon)\right) \alpha_{n},
\end{aligned}
$$

and Eq. (A6) into

$$
\begin{aligned}
& \left(R^{n}-\frac{\omega^{2}}{\omega^{2}+n^{2}}\left(1-\frac{\epsilon}{\pi}\right)(R-a)^{n}\right) \alpha_{n}= \\
& -\frac{2}{\pi n}\left(\alpha_{0}-\frac{R^{2}}{4 D_{2}}+T\right)\left(\frac{\omega^{2}}{\omega^{2}+n^{2}} \sin (n \epsilon)+\frac{n \omega}{\omega^{2}+n^{2}} \tanh (\omega(\pi-\epsilon)) \cos (n \epsilon)\right) \\
& -\frac{2 \omega^{2}}{\pi\left(\omega^{2}+n^{2}\right)} \sum_{m=1}^{\infty} \alpha_{m} \frac{(R-a)^{m}}{\omega^{2}+m^{2}} \cos (m \epsilon)(\omega \cos (n \epsilon) \tanh (\omega(\pi-\epsilon))-n \sin (n \epsilon)) \\
& -\frac{\omega^{2}}{\pi}\left(\sum_{m \neq n} \frac{\alpha_{m}}{\omega^{2}+m^{2}}(R-a)^{m} \frac{\sin ((m-n) \epsilon)}{m-n}+\sum_{m=1}^{\infty} \frac{\alpha_{m}}{\omega^{2}+m^{2}}(R-a)^{m} \frac{\sin ((m+n) \epsilon)}{m+n}\right) .
\end{aligned}
$$

\section{Particular case $\lambda=0$}

In this case, the previous equations can be solved exactly, leading to

$$
\alpha_{0}-\frac{R^{2}}{4 D_{2}}=\frac{1}{3} \frac{R^{2}}{D_{1}} \frac{(\pi-\epsilon)^{3}}{\pi}
$$

and

$$
\alpha_{n}=-\frac{2}{\pi} \frac{R^{2}}{D_{1}} \frac{n(\pi-\epsilon) \cos (n \epsilon)+\sin (n \epsilon)}{n^{3}} \frac{1}{R^{n}} .
$$

We note that the particular case $a=0$ is also described by these expressions, although it does not seem to be clear from Eqs. (A5)-(A6).

\section{Particular case $a=R$}

Here again, Eqs. (A5)- (A6) can be solved exactly, and give :

$$
\alpha_{0}-\frac{R^{2}}{4 D_{2}}=\left(\frac{1}{\lambda}+\frac{R^{2}}{4 D_{2}}\right) \frac{1-\frac{\epsilon}{\pi}-\frac{\tanh (\omega(\pi-\epsilon))}{\pi \omega}}{\frac{\epsilon}{\pi}+\frac{\tanh (\omega(\pi-\epsilon))}{\pi \omega}},
$$


and

$$
\alpha_{n}=-\frac{2}{\pi}\left(\frac{1}{\lambda}+\frac{R^{2}}{4 D_{2}}\right) \frac{\omega}{\omega^{2}+n^{2}} \frac{\frac{\omega}{n} \sin (n \epsilon)+\tanh (\omega(\pi-\epsilon)) \cos (n \epsilon)}{\frac{\epsilon}{\pi}+\frac{\tanh (\omega(\pi-\epsilon))}{\pi \omega}} \frac{1}{R^{n}} .
$$

\section{Perturbative approach}

Expanding $\alpha_{0}$ and $\alpha_{n}$ in powers of $\epsilon$

$$
\alpha_{0}=\alpha_{0}^{(0)}+\alpha_{0}^{(1)} \epsilon+\alpha_{0}^{(2)} \epsilon^{2}+\ldots \text { and } \alpha_{n}=\alpha_{n}^{(0)}+\alpha_{n}^{(1)} \epsilon+\alpha_{n}^{(2)} \epsilon^{2}+\ldots
$$

Eqs. (A5)-(A6) lead, after lengthy calculations, to

$$
\begin{aligned}
& \alpha_{0}=\frac{R^{2}}{4 D_{2}}+\omega^{2} T\left\{\left(2 \sum_{m=1}^{\infty} \frac{1}{\omega^{2}\left(1-x^{m}\right)+m^{2}}\right)-\pi \epsilon+\left(1+2 \omega^{2} \sum_{m=1}^{\infty} \frac{1-x^{m}}{\omega^{2}\left(1-x^{m}\right)+m^{2}}\right) \epsilon^{2}\right\}+\ldots, \\
& \alpha_{n}=\frac{\omega^{2} T}{R^{n}\left(\omega^{2}\left(1-x^{n}\right)+n^{2}\right)}\left\{-2+n^{2} \epsilon^{2}+\ldots\right\} .
\end{aligned}
$$

\section{Appendix B: A second integral equation satified by $t_{1}$ in the $2 \mathrm{D}$ case}

Using Eq. (7) for the Fourier coefficients in Eq. (A4) leads to a second integral equation satisfied by $t_{1}$

$$
t_{1}(\theta)=T\left(1-\frac{\cosh (\omega(\pi-\theta))}{\cosh (\omega(\pi-\epsilon))}\right)+\int_{\epsilon}^{2 \pi-\epsilon} t_{1}(\alpha)\left(J(\theta, \alpha)-\frac{\cosh (\omega(\pi-\theta))}{\cosh (\omega(\pi-\epsilon))} J(\epsilon, \alpha)\right) \mathrm{d} \alpha
$$

where

$$
J(\theta, \alpha) \equiv \frac{1}{2 \pi}+\frac{1}{\pi} \sum_{n=1}^{\infty} \frac{\omega^{2}}{\omega^{2}+n^{2}}\left(1-\frac{a}{R}\right)^{n} \cos (n \theta) \cos (n \alpha)
$$

This equation is especially well adapted to local expansions of $t_{1}(\theta)$ in the vicinity of $a \simeq R$, but it can also be rearranged into the following integral equation, useful when $a \ll R$ :

$$
\begin{aligned}
t_{1}(\theta) & =T\left(1-\frac{\cosh (\omega(\pi-\theta))}{\cosh (\omega(\pi-\epsilon))}\right)+\frac{\omega}{\sinh (2 \omega(\pi-\epsilon))} \int_{\epsilon}^{2 \pi-\epsilon} t_{1}\left(\theta^{\prime}\right) \sinh \left(\omega\left(\theta_{<}-\epsilon\right)\right) \sinh \left(\omega\left(2 \pi-\epsilon-\theta_{>}\right)\right) \mathrm{d} \theta^{\prime}+ \\
& +\int_{\epsilon}^{2 \pi-\epsilon} t_{1}(\alpha)\left(\widetilde{J}(\theta, \alpha)-\frac{\cosh (\omega(\pi-\theta))}{\cosh (\omega(\pi-\epsilon))} \widetilde{J}(\epsilon, \alpha)\right) \mathrm{d} \alpha,
\end{aligned}
$$

where

$$
\widetilde{J}(\theta, \alpha) \equiv \frac{1}{\pi} \sum_{n=1}^{\infty} \frac{\omega^{2}}{\omega^{2}+n^{2}}\left(\left(1-\frac{a}{R}\right)^{n}-1\right) \cos (n \theta) \cos (n \alpha)
$$

\section{Appendix C: Computation of $I_{\epsilon}(m, n)$ in 3D}

In this Appendix, we provide the explicit formula for the matrix $I_{\epsilon}(m, n)$ in 3D case. Although technical, this is an important result for a numerical computation because it allows one to avoid an approximate integration in Eq. (64) which otherwise could be a significant source of numerical errors. The formula (C6) for non-diagonal elements is somewhat elementary, while the derivation for diagonal elements seems to be original. 


\section{Non-diagonal elements}

The Legendre polynomials satisfy

$$
\frac{d}{d x}\left[\left(1-x^{2}\right) \frac{d}{d x} P_{n}(x)\right]+n(n+1) P_{n}(x)=0
$$

from which

$$
\int_{a}^{b} d x P_{n}(x)=-\frac{\left[\left(1-x^{2}\right) P_{n}^{\prime}(x)\right]_{a}^{b}}{n(n+1)} \quad(n>0) .
$$

and

$$
\int_{a}^{b} d x P_{m}(x) P_{n}(x)=\frac{\left[\left(1-x^{2}\right)\left[P_{m}(x) P_{n}^{\prime}(x)-P_{n}(x) P_{m}^{\prime}(x)\right]\right]_{a}^{b}}{m(m+1)-n(n+1)} \quad(m \neq n) .
$$

Since

$$
\left(1-x^{2}\right) P_{n}^{\prime}(x)=-n x P_{n}(x)+n P_{n-1}(x)=(n+1) x P_{n}(x)-(n+1) P_{n+1}(x)
$$

we find

$$
\int_{a}^{b} d x P_{n}(x)=\frac{\left[x P_{n}(x)-P_{n-1}(x)\right]_{a}^{b}}{n+1} \quad(n>0)
$$

and

$$
\int_{a}^{b} d x P_{m}(x) P_{n}(x)=\frac{\left.\left[(m-n) x P_{m}(x) P_{n}(x)+n P_{n-1}(x) P_{m}(x)-m P_{m-1}(x) P_{n}(x)\right]\right]_{a}^{b}}{m(m+1)-n(n+1)} \quad(m \neq n) .
$$

From the above formulas, we get

$$
\begin{aligned}
I_{\epsilon}(m, n) & =m \frac{(n-m) u P_{m}(u) P_{n}(u)+(m+1) P_{m}(u) P_{n-1}(u)-(n+1) P_{n}(u) P_{m-1}(u)}{(n+1)[m(m+1)-n(n+1)]}, \\
u & =\cos \epsilon \quad(m \neq n) .
\end{aligned}
$$

\section{Diagonal elements}

We denote

$$
K_{n}=\int_{a}^{b} d x P_{n}^{2}(x)
$$

Using the relation

$$
P_{n}(x)=\frac{2 n-1}{n} x P_{n-1}(x)-\frac{n-1}{n} P_{n-2}(x),
$$

we obtain

$$
K_{n}=\frac{2 n-1}{n} \int_{a}^{b} d x x P_{n-1}(x) P_{n}(x)-\frac{n-1}{n} \int_{a}^{b} d x P_{n-2}(x) P_{n}(x)
$$


The second integral is given by Eq. ([C6). In order to compute the first one, we consider

$$
\begin{aligned}
0 & =\int_{a}^{b} d x\left\{x P_{n-1}(x)\left[\frac{d}{d x}\left[\left(1-x^{2}\right) \frac{d}{d x} P_{n}(x)\right]+n(n+1) P_{n}(x)\right]\right. \\
& \left.-x P_{n}(x)\left[\frac{d}{d x}\left[\left(1-x^{2}\right) \frac{d}{d x} P_{n-1}(x)\right]+(n-1) n P_{n-1}(x)\right]\right\} \\
& =2 n \int_{a}^{b} d x x P_{n-1}(x) P_{n}(x)+\left[x P_{n-1}(x)\left(1-x^{2}\right) P_{n}^{\prime}(x)-x P_{n}(x)\left(1-x^{2}\right) P_{n-1}^{\prime}(x)\right]_{a}^{b} \\
& -\int_{a}^{b} d x\left(1-x^{2}\right)\left[P_{n}^{\prime}(x) P_{n-1}(x)-P_{n-1}^{\prime}(x) P_{n}(x)\right] .
\end{aligned}
$$

The last integral can be written as

$$
\begin{aligned}
J & =\int_{a}^{b} d x\left(1-x^{2}\right) P_{n}^{2}(x)\left(P_{n-1}(x) / P_{n}(x)\right)^{\prime}=\left[\left(1-x^{2}\right) P_{n-1}(x) P_{n}(x)\right]_{a}^{b} \\
& -\int_{a}^{b} d x\left(P_{n-1}(x) / P_{n}(x)\right)\left[-2 x P_{n}^{2}(x)+2\left(1-x^{2}\right) P_{n}^{\prime}(x) P_{n}(x)\right] \\
& =\left[\left(1-x^{2}\right) P_{n-1}(x) P_{n}(x)\right]_{a}^{b}-2 \int_{a}^{b} d x\left[-x P_{n-1}(x) P_{n}(x)+\left(1-x^{2}\right) P_{n}^{\prime}(x) P_{n-1}(x)\right] .
\end{aligned}
$$

In the last term, we substitute $\left(1-x^{2}\right) P_{n}^{\prime}(x)$ to get

$$
J=\left[\left(1-x^{2}\right) P_{n-1}(x) P_{n}(x)\right]_{a}^{b}+2 \int_{a}^{b} d x x P_{n-1}(x) P_{n}(x)-2 \int_{a}^{b} d x\left[-n x P_{n}(x)+n P_{n-1}(x)\right] P_{n-1}(x) .
$$

Bringing these results together, we get

$$
\begin{aligned}
0 & =2 n \int_{a}^{b} d x x P_{n-1}(x) P_{n}(x)+\left[x P_{n-1}(x)\left(1-x^{2}\right) P_{n}^{\prime}(x)-x P_{n}(x)\left(1-x^{2}\right) P_{n-1}^{\prime}(x)\right]_{a}^{b} \\
& +\left[\left(1-x^{2}\right) P_{n-1}(x) P_{n}(x)\right]_{a}^{b}+2 \int_{a}^{b} d x x P_{n-1}(x) P_{n}(x)-2 \int_{a}^{b} d x\left[-n x P_{n}(x)+n P_{n-1}(x)\right] P_{n-1}(x)
\end{aligned}
$$

so that

$$
\int_{a}^{b} d x x P_{n-1}(x) P_{n}(x)=\frac{-1}{4 n+2}\left[x\left(1-x^{2}\right)\left[P_{n-1}(x) P_{n}^{\prime}(x)-P_{n}(x) P_{n-1}^{\prime}(x)\right]+\left(1-x^{2}\right) P_{n-1}(x) P_{n}(x)\right]_{a}^{b}+\frac{n}{2 n+1} K_{n-1} .
$$

We obtain

$$
\begin{aligned}
K_{n} & =-\frac{2 n-1}{2 n(2 n+1)}\left[x\left(1-x^{2}\right)\left[P_{n-1}(x) P_{n}^{\prime}(x)-P_{n}(x) P_{n-1}^{\prime}(x)\right]+\left(1-x^{2}\right) P_{n-1}(x) P_{n}(x)\right]_{a}^{b}+\frac{2 n-1}{2 n+1} K_{n-1} \\
& -\frac{n-1}{n}\left[\frac{2 x P_{n-2}(x) P_{n}(x)-n P_{n-1}(x) P_{n-2}(x)+(n-2) P_{n-3}(x) P_{n}(x)}{2(2 n-1)}\right]_{a}^{b} .
\end{aligned}
$$


We can further simplify this expression by using the following identities

$$
\begin{aligned}
& (n-1) P_{n-1}(x)-(2 n-3) x P_{n-2}(x)+(n-2) P_{n-3}(x)=0, \\
& n P_{n}(x)-(2 n-1) x P_{n-1}(x)+(n-1) P_{n-2}(x)=0, \\
& \left(1-x^{2}\right) P_{n}^{\prime}(x)=-n x P_{n}(x)+n P_{n-1}(x), \\
& \left(1-x^{2}\right) P_{n-1}^{\prime}(x)=n x P_{n-1}(x)-n P_{n}(x) .
\end{aligned}
$$

We get

$$
\begin{aligned}
K_{n} & =-\frac{2 n-1}{2 n(2 n+1)}\left[n x\left[P_{n-1}^{2}(x)+P_{n}^{2}(x)-2 x P_{n}(x) P_{n-1}(x)\right]+\left(1-x^{2}\right) P_{n-1}(x) P_{n}(x)\right]_{a}^{b}+\frac{2 n-1}{2 n+1} K_{n-1} \\
& -\frac{n-1}{2 n(2 n-1)}\left[(2 n-1) x P_{n}(x) P_{n-2}(x)-n P_{n-1}(x) P_{n-2}(x)-(n-1) P_{n-1}(x) P_{n}(x)\right]_{a}^{b} \\
& =-\frac{2 n-1}{2 n(2 n+1)}\left[n x\left[P_{n-1}^{2}(x)+P_{n}^{2}(x)-2 x P_{n}(x) P_{n-1}(x)\right]+\left(1-x^{2}\right) P_{n-1}(x) P_{n}(x)\right]_{a}^{b}+\frac{2 n-1}{2 n+1} K_{n-1} \\
& -\frac{1}{2 n}\left[\left((2 n-1) x^{2}+1\right) P_{n}(x) P_{n-1}(x)-n x\left(P_{n-1}^{2}(x)+P_{n}^{2}(x)\right)\right]_{a}^{b} \\
& =\frac{\left[x\left(P_{n-1}^{2}(x)+P_{n}^{2}(x)\right)-2 P_{n}(x) P_{n-1}(x)\right]_{a}^{b}}{2 n+1}+\frac{2 n-1}{2 n+1} K_{n-1}
\end{aligned}
$$

and we know that $K_{0}=b-a$. Applying this formula recursively, one finds

$$
K_{n}=\frac{F_{n}(b)-F_{n}(a)}{2 n+1},
$$

where

$$
\begin{aligned}
F_{n}(x) & =x\left[P_{n}^{2}(x)+2 P_{n-1}^{2}(x)+\ldots+2 P_{1}^{2}(x)+P_{0}(x)\right] \\
& -2 P_{n}(x) P_{n-1}(x)-2 P_{n-1}(x) P_{n-2}(x)-\ldots-2 P_{1}(x) P_{0}(x)+x \\
& =\sum_{k=1}^{n}\left[2(x-1) P_{k}^{2}(x)+\left[P_{k}(x)-P_{k-1}(x)\right]^{2}\right]-(x-1) P_{n}^{2}(x)+(x-1) P_{0}^{2}(x)+x .
\end{aligned}
$$

One can check that this function satisfies the recurrent relation

$$
F_{n}(x)=F_{n-1}(x)+x\left[P_{n}^{2}(x)+P_{n-1}^{2}(x)\right]-2 P_{n}(x) P_{n-1}(x), \quad F_{0}(x)=x .
$$

Note that $F_{n}( \pm 1)=F_{n-1}( \pm 1)=\ldots= \pm 1$.

As a result, we obtain

$$
I_{\epsilon}(n, n)=-P_{n}(u) \frac{u P_{n}(u)-P_{n-1}(u)}{n+1}+\frac{F_{n}(u)+1}{2 n+1}, \quad u=\cos \epsilon
$$

[1] S. A. Rice, Diffusion-limited reactions, vol. 25 (Elsevier, Amsterdam, 1985).

[2] P. Hänggi, P. Talkner, and M. Borkovec, Reviews of Modern Physics 62 (1990).

[3] B. Alberts, A. Johnson, J. Lewis, M. Raff, K. Roberts, and P. Walter, Molecular Biology of the Cell (Garland New York, 2002).

[4] O. Bénichou et al., Phys Rev Lett 94, 198101 (2005); J Phys Condens Matter 17, S4275 (2005)

[5] O. Bénichou, C. Loverdo, M. Moreau, and R. Voituriez, Physical Chemistry Chemical Physics 10, 7059 (2008).

[6] M. Coppey, O. Bénichou, R. Voituriez, and M. Moreau, Biophys. J. 87, 1640 (2004).

[7] O. G. Berg, R. B. Winter, and P. H. von Hippel, Biochemistry 20, 6929 (1981).

[8] M. Slutsky and L. A. Mirny, Biophysical Journal 87, 4021 (2004).

[9] I. Eliazar, T. Koren, and J. Klafter, Journal of Physics: Condensed Matter 19 (2007).

[10] M. A. Lomholt, T. Ambjornsson, and R. Metzler, Phys. Rev. Lett. 95, 260603 (2005). 
[11] O. Bénichou, M. Coppey, M. Moreau, P. H. Suet, and R. Voituriez, EPL (Europhysics Letters) 70, 42 (2005).

[12] P. Levitz et al., Phys Rev Lett 96, 180601 (2006); Phys Rev E 78, 030102 (2008).

[13] A. V. Chechkin, I. M. Zaid, M. A. Lomholt, I. M. Sokolov, and R. Metzler, Physical Review E 79 (2009).

[14] C. Loverdo, O. Bénichou, R. Voituriez, A. Biebricher, I. Bonnet, and P. Desbiolles, Physical Review Letters 102, 188101 (2009).

[15] G. Adam and M. Delbrück, Reduction of dimensionality in biological diffusion processes (W.H. Freeman Co, Publishers, San Francicso, 1968).

[16] H. Sano and M. Tachiya, The Journal of Chemical Physics 75, 2870 (1981).

[17] R. D. Astumian and P. B. Chock, The Journal of Physical Chemistry 89, 3477 (1985).

[18] G. C. Bond, Heterogeneous Catalysis: Principles and Applications (Clarendon, Oxford, 1987).

[19] A. Blumen, G. Zumofen, and J. Klafter, Physical Review B 30 (1984)

[20] Z. Schuss, A. Singer, and D. Holcman, Proceedings of the National Academy of Sciences 104, 16098 (2007).

[21] J. J. Kozak and V. Balakrishnan, Physical Review E 65 (2002).

[22] S. Condamin et al., Nature 450, 77 (2007); O. Bénichou, B. Meyer, V. Tejedor, and R. Voituriez, Phys Rev Lett 101, 130601 (2008); V. Tejedor, O. Bénichou and R. Voituriez, Phys Rev E 80, 065104 (2009).

[23] E. Agliari, Physical Review E (Statistical, Nonlinear, and Soft Matter Physics) 77, 011128 (2008).

[24] C. P. Haynes and A. P. Roberts, Physical Review E (Statistical, Nonlinear, and Soft Matter Physics) 78, 041111 (2008).

[25] S. Reuveni, R. Granek, and J. Klafter, Physical Review E 81, 040103 (2010).

[26] S. Redner, A guide to first-passage processes (Cambridge University Press, Cambridge, England, 2001).

[27] S. Condamin, O. Bénichou, and M. Moreau, Phys Rev Lett 95, 260601 (2005); Phys Rev E 75, 021111 (2007).

[28] D. S. Grebenkov, Physical Review E (Statistical, Nonlinear, and Soft Matter Physics) 76, 041139 (2007).

[29] S. Condamin, O. Bénichou, and J. Klafter, Phys Rev Lett 98, 250602 (2007).

[30] A. Singer, Z. Schuss, and D. Holcman, Journal of Statistical Physics 122, 465 (2006).

[31] S. Pillay, M. J. Ward, A. Peirce, and T. Kolokolnikov, Multiscale Modeling \& Simulation 8, 803 (2010).

[32] A. F. Cheviakov, M. J. Ward, and R. Straube, Multiscale Modeling \& Simulation 8, 836 (2010).

[33] O. Bénichou and R. Voituriez, Physical Review Letters 100, 168105 (2008).

[34] G. Oshanin, M. Tamm, and O. Vasilyev, The Journal of Chemical Physics 132, 235101 (2010).

[35] C. Chevalier, O. Bénichou, B. Meyer, and R. Voituriez, Journal of Physics A: Mathematical and Theoretical 44, 025002 (2011).

[36] I. V. Grigoriev, Y. A. Makhnovskii, A. M. Berezhkovskii, and V. Y. Zitserman, The Journal of Chemical Physics 116, 9574 (2002).

[37] O. Bénichou, D. Grebenkov, P. Levitz, C. Loverdo, and R. Voituriez, Physical Review Letters 105, 150606 (2010).

[38] A. Singer, Z. Schuss, D. Holcman, and R. Eisenberg, Journal of Statistical Physics 122, 437 (2006).

[39] G. Barton, Elements of Green's Functions and Propagation (Oxford Science Publications, 1989). 